\title{
The High Energy Frontier
}

\author{
Carlo Rubbia
}

CERN

\begin{abstract}
This is a review of the present status of particle physics and the main scientific goals in our work at the "High Energy Frontier" with specific emphasis on connections to Cosmology. Based on an extraordinarily successful Standard Model, our field is exploring the fundamental questions such as the nature of mass and the unification scheme which only a few decades ago seemed to belong mostly to the realm of dreams. Neutrino masses and oscillations, the key to CP-violation and proton decay are actively searched for at accelerators and underground experiments.
\end{abstract}

\section{The role of Particle Physics in Astrophysics}

The purpose of particle physics is to explore and understand the deepest structure of matter. The resolution available with our present instruments is today about $10^{-18}$ metres. Quantum Mechanics implies that the price to pay for such high resolution is high colliding energy. The energy reached at the constituent level is presently of the order of $100 \mathrm{GeV}$, but the next generation accelerator in preparation, the Large Hadron Collider (LHC), will reach the 1 $\mathrm{TeV}$ level which corresponds to a resolution of $10^{-19}$ metres.

There is a complementarity between particle physics and telescope observations (Astronomy). The basic dividing line is the moment at which the universe became transparent to light. This particular instant of the life of our universe, about 300,000 years after the Big Bang, is studied by satellite observations, such as $\mathrm{COBE}$, measuring the fluctuations of the $3 \mathrm{~K}$ microwave radiation remnant of the radiation emitted at that time. Accelerators can reproduce on a very tiny scale (but in the Laboratory!) conditions which were typical for the whole early universe. It is the remarkable uniformity of the universe which makes then such studies relevant! 
Particle physics can also provide information on invisible particles such as neutrinos and on the interior of celestial bodies (sun, neutron stars etc.), and, more generally, whenever direct observations are impossible.

One can say that particle physics provides the basic knowledge on particles and forces acting in the universe, extending and completing the role of general relativity in predicting and explaining cosmic phenomena such as the Big Bang, supernovae (SN1987A), etc.

\section{The present framework: quarks, leptons and basic interactions}

The spectrum of elementary fermions appears today as firmly and definitely established with the well known three families (Figure 1). With the, now presumed correct, recent observation of the top quark [1] at Fermilab all elements of the quark family have been observed.

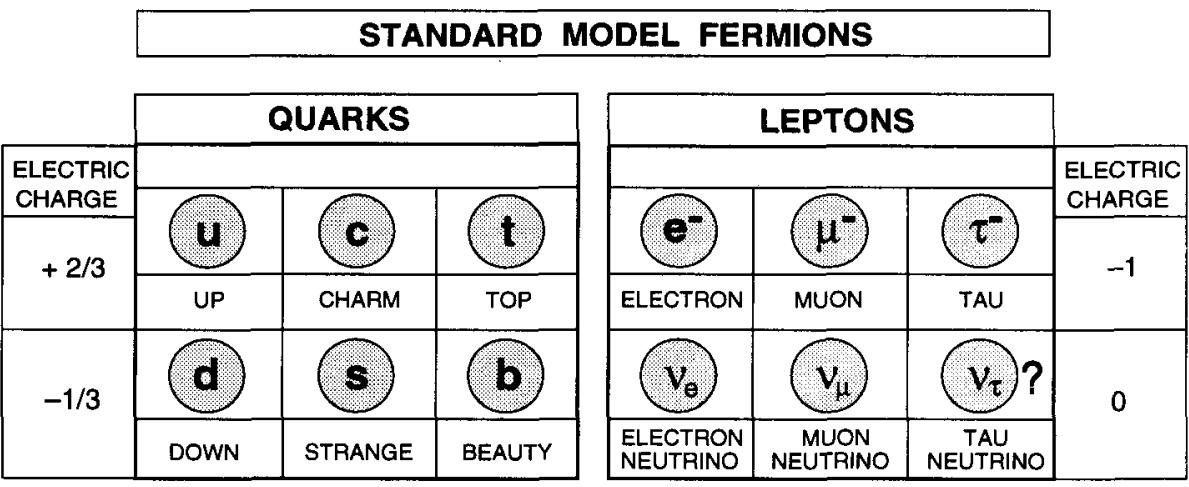

3 colours

Figure 1: Table of Standard Model quarks and leptons including some of their main properties.

There are six quarks, set up in three families, each one grouping two quarks differing by one unit of charge. In parallel with these three quark families, we have three families of leptons containing each a negatively charged particle and a neutrino. Each quark exists under three varieties of "colour", which we can dub as "red", "green" and "blue", whereas the leptons have no "colour". This altogether represents 24 fundamental particles, to which one associates as many antiparticles, with the same masses but the opposite charges and "colours". 


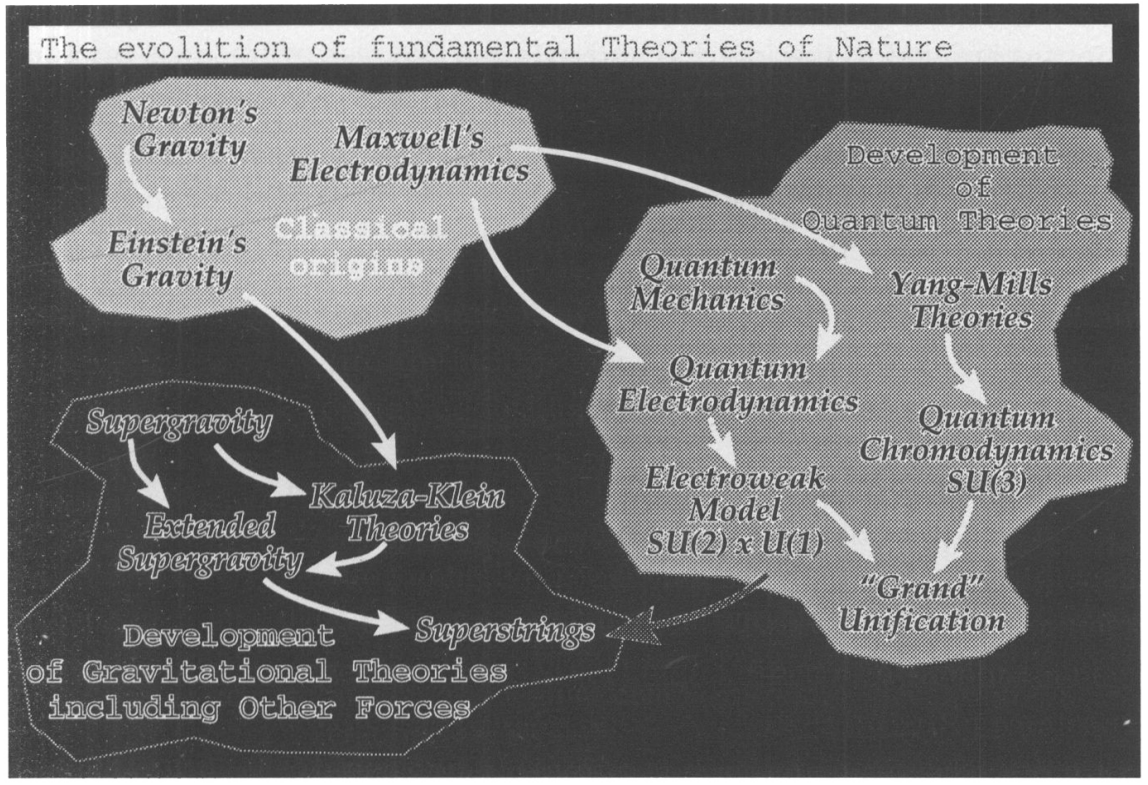

Figure 2: :Summary of the evolution of the fundamental theories of nature.

LEP has firmly set to three the number of Neutrino species. Even if the $v_{\tau}$ has not been "directly" and conclusively detected, it is assumed to exist. The postulated basic symmetry between quarks and leptons tells us that the three known generations complete the picture.

This is a lot of fundamental particles ! However, we have long realised that, at this level of exploration, unity and simplicity should not be looked for in a very small number of fundamental fields. Indeed, in the framework of superstring theory, which we think would reveal itself openly at the level of 10-35 metre, all these fermion fields appear as the many lowest excitation modes of a superstring, which we can visualise as a tiny loop closed upon itself at the level of $10^{-35}$ metre, and probably in more space dimensions than our usual three dimensions, [the other ones being compactified at the level of 10-35 metre]. Much unity and simplicity is found, however, but it is in the form which all interactions take at the quark and lepton level. Unity and simplicity are thus found in the scripts which the actors follow far more than in the numerous cast of characters.

Theories are evolving from a classical origin towards a unified theory of all interactions based on quantum mechanics and relativity, via somewhat intricate paths (Figure 2). In analogy with General Relativity, the origin of all 
basic interactions is found in "geometrical" properties, i.e. in local gauge symmetries applied to the internal and external degrees of freedom of quarks and leptons. They determine uniquely the nature and structure of their interactions. Lorentz invariance and specific gauge symmetries thus specify the nature and structure of all these interactions. The basic processes all correspond to the exchange of a quantum of the gauge field (Figure 3) and the number of gauge fields is specified in each case by the gauge symmetry group:

- the electro-weak theory is based on the $\mathrm{SU}(2) \times \mathrm{U}(1)$ group structure and the exchange of a photon, of a charged $\mathrm{W}^{ \pm}$and of a neutral $Z^{0}$.

- the QCD theory describing the strong interaction is based on the $\mathrm{SU}(3)$ group structure and the exchange of $\mathbf{8}$ gluons each carrying "colour".

All the gauge fields correspond to vector particles of spin 1 . There is therefore a great unity and a great simplicity at the level of the basic interactions! The possible existence of such a general and simple principle at the origin of all forces was realised twenty years ago. Since then we have found the experimental proofs that Nature does behave that way. We have reached a good understanding of the deep origin and of the nature of forces.

\section{A complicated vacuum}

Yet, there are some twists to this simple picture. In a gauge theory, the gauge fields should all have a zero mass. This is the case for the photon and for the gluons but the $\mathrm{W}$ and the $\mathrm{Z}$ are very massive, with masses close to a hundred times that of the proton. The strict invariance property used also seems to require all quarks and leptons to be massless, which is obviously not the case. The answer to this problem is however provided by the Higgs mechanism. It allows a breaking of the primordial symmetry, providing masses to the particles but keeping enough relations untouched so that the theory remains renormalizable.

The blame for the apparent lack of symmetry is thus put on the vacuum. The vacuum, which corresponds by definition to the lowest energy state, contains a field with a non-zero expectation value, the Higgs field. Fluctuation quanta from this non-zero expectation value should correspond to the presence of a neutral spin zero particle, the Higgs meson, but its mass remains an open 

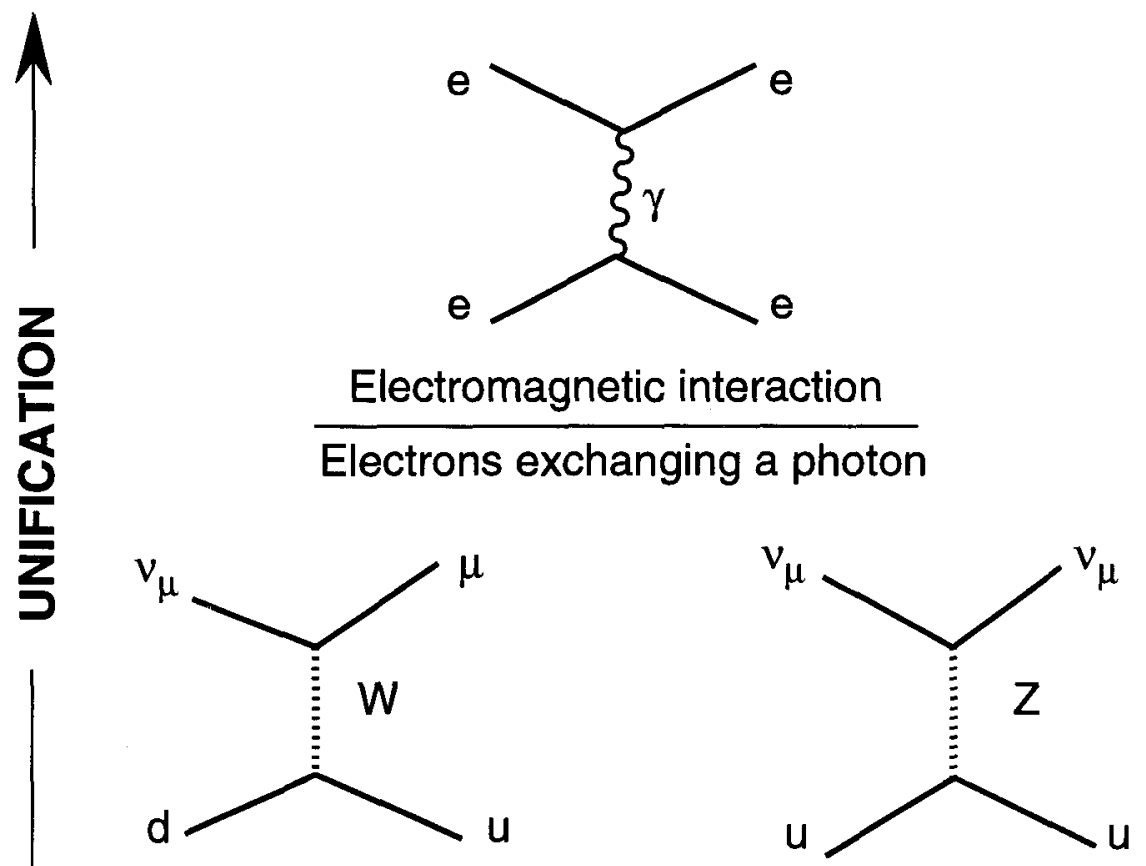

\section{Weak interaction}

Quark and lepton exchanging a W or a Z

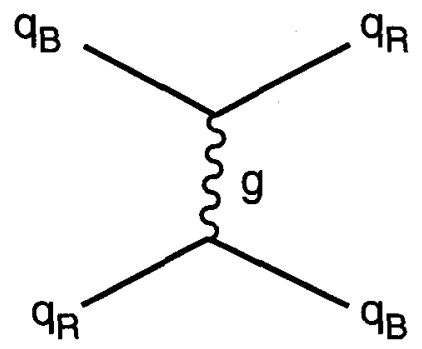

\section{Strong interaction}

\section{Quarks exchanging a gluon and colour}

Figure 3 The Standard Model interactions : at the top, Feynman graphs where a photon, a $W$ boson and a $Z$ boson are exchanged as examples of the electroweak interaction and at the bottom, a Feynman graph where a gluon is exchanged as an example of the strong interaction. 
parameter in the theory. As a consequence of the presence of the Higgs field, the vacuum behaves like a medium with properties which are very similar to those of a superconductor. Indeed, one may say that the $\mathrm{W}$ and the $\mathrm{Z}$ appear with large masses in the vacuum in much the same way as a photon acquires a mass inside a superconductor.

There is yet another twist. Whereas the gluon has a zero mass and should, as the photon does, give rise to an interaction of infinite range, the strong interaction has a finite range. This is now due to the fact that the gluon carries "colour" and that the vacuum is opaque to "colour", with a penetration length of the order of one fermi. It is this penetration length which specifies the finite range of the strong interaction. One may say that the vacuum behaves again like a superconducting medium with respect to "colour".

These peculiar properties of the vacuum represent one of the great challenges of particle physics today. We have a beautiful and simple formulation for all the forces but it operates in a complicated vacuum. One may say that with the Glashow-Salam-Weinberg (G-S-W) electroweak theory we are at the level of the Landau-Ginsburg theory of superconductivity. The G-S-W theory is of course relativistic and non Abelian, but the basic idea is similar. We have to realise that we have not yet reached the Bardeen-Cooper-Schrieffer level, with an understanding of the dynamics behind symmetry breaking. We do not know yet what the nature of the Higgs field is. Is it an elementary field? Is it a bound state of hitherto unknown fermions? This is one of the key questions today and it should bring us to the fundamental origin of mass. We can estimate the critical temperature at which the vacuum would return to normal and the masses of the $\mathrm{W}$ and of the $\mathrm{Z}$ would disappear. It is of the order of 200 $\mathrm{GeV}$. The phase transition should therefore have occurred when our universe was about $10^{-11}$ second old. We are led to expect dramatic new features as we reach collision energies of the order of $1 \mathrm{TeV}$ and beyond, that is at collision energies neatly above that corresponding to the critical electroweak temperature. This is the energy scale which defines the next big step, corresponding to the LHC in preparation at CERN, the European laboratory for particle physics.

For "colour" we expect the vacuum to turn from superconducting to normal and become transparent to "colour" when the temperature reaches a level of the order of $200 \mathrm{MeV}$. This transition should have occurred when the universe was a little over 10 microseconds old. This is what we are trying to reach at CERN with relativistic heavy ion collisions (Figure 4), a programme which benefits 
from a newly installed lead source in operation at the SPS and which will cover another tremendous step forward with the LHC.

Granting the existence of these challenging complications associated with the structure of our too cold vacuum (much cooler than the critical temperature just mentioned above), physics at the quark and lepton level is particularly simple. This is the Standard Model of fundamental particles and fundamental interactions which provides our understanding of the dynamics at the deepest accessible level today.

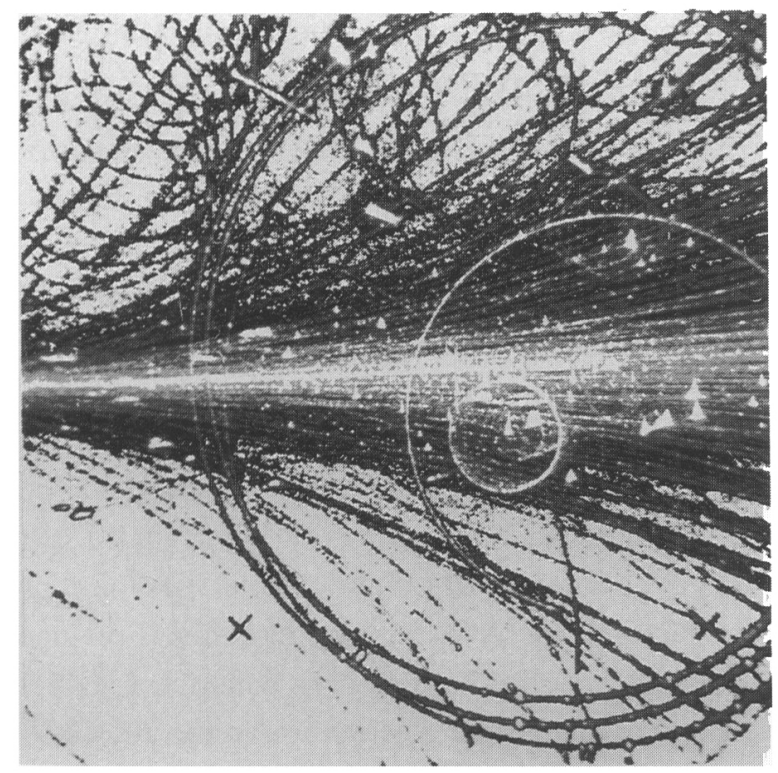

Figure 4: Example of a heavy ion collision at the CERN SPS. This is a 200 $\mathrm{GeV} /$ nucleon Sulphur + Sulphur collision observed by the Na-35 Collaboration.

\section{The Standard Model}

Twenty years ago the Standard Model appeared as an interesting possibility but it did not have only supporters. Its renormalizability and its "asymptotic freedom" have long fuelled the enthusiasm of the theorists and, over the past twenty years, many striking experimental results have beautifully vindicated 
it. The two key ones came from CERN, namely the discovery of the "neutral current" interaction in 1973 [2], and the discovery of the $W$ [3] and Z [4] bosons in 1983. At the same time the $\bar{p}$ collider and now LEP have allowed us to obtain many important results on jets which are the way quarks and gluons manifest themselves most openly in a vacuum opaque to "colour".

A gauge theory specifies the form of the coupling but not the value of the coupling constant. There are two independent couplings for the electroweak theory since the gauge group is $\mathrm{SU}(2) \times \mathrm{U}(1)$. There is only one for the strong force, which is based on the $\mathrm{SU}(3)$ gauge group. They vary in a predictable way with the momentum transfer squared at which the process occurs or, more generally, with the energy scale at which a process is analysed. The coupling constants "run". This has also been verified.

The close similarities between the electroweak and strong couplings, together with the fact that the three couplings approach one another with increasing energy to eventually meet for a value which the LEP results now lead us to expect to be around $10^{16} \mathrm{GeV}$ [5], strongly suggests a Grand Unified Theory (GUT) where quarks and leptons would appear as different manifestations of the same field. A GUT mixes quarks and leptons and this implies proton decay, a process which we should be very much concerned with. We do not know yet the form which such a GUT takes. This is also one of the great questions today. Superstrings have been a guide line in our thinking in that direction for almost a decade. They however refer to the Planck scale, $10^{19} \mathrm{GeV}$, where gravity fully comes into the picture. We have not yet succeeded, however, in extracting from such a "Theory of Everything" compelling constraints which would apply to our present level of experimental investigation in the $100 \mathrm{GeV}$ domain.

The Standard Model has so far successfully passed all tests with flying colours. It works so well that one has become impatient to find some deviations heralding new physics but nothing has appeared so far. The success of the Standard Model can be illustrated by many results. Let us look at but a few. As we have mentioned already the electroweak theory has two couplings. One can be chosen as the electromagnetic coupling $\alpha$. The coupling constant $\alpha$ at LEP is not the standard fine structure constant $\alpha$, which is defined at zero momentum transfer and takes the well known value of 1/137.0459895(61). It should be an $\alpha$ "having run" to the mass of the $Z$. One finds indeed a value of $1 / 128.87(12)[6]$. The strength of the coupling has increased as it should have done. The other coupling can be conveniently defined in terms of the Weinberg angle $\theta_{\mathrm{W}}$, and the quantity $\sin ^{2} \theta \mathrm{W}$ specifies, for instance, how the neutral current coupling ( $\mathrm{Z}$ 
exchange) relates to the standard charged one ( $\mathrm{W}$ exchange) in the Born approximation. The same value of this parameter should be extracted from many different independent results if, as the electroweak theory claims, it is enough to specify all these processes. This turns out to be the case (Figure 5).

To illustrate the universality of the weak coupling one can also compare the vector and axial vector couplings measured at LEP [7] (that is in the time-like region with lepton-antilepton annihilation) with their values extracted from a recent experiment on neutrino-electron scattering [8] (that is in the space-like region with lepton-lepton scattering) (Figure 6).

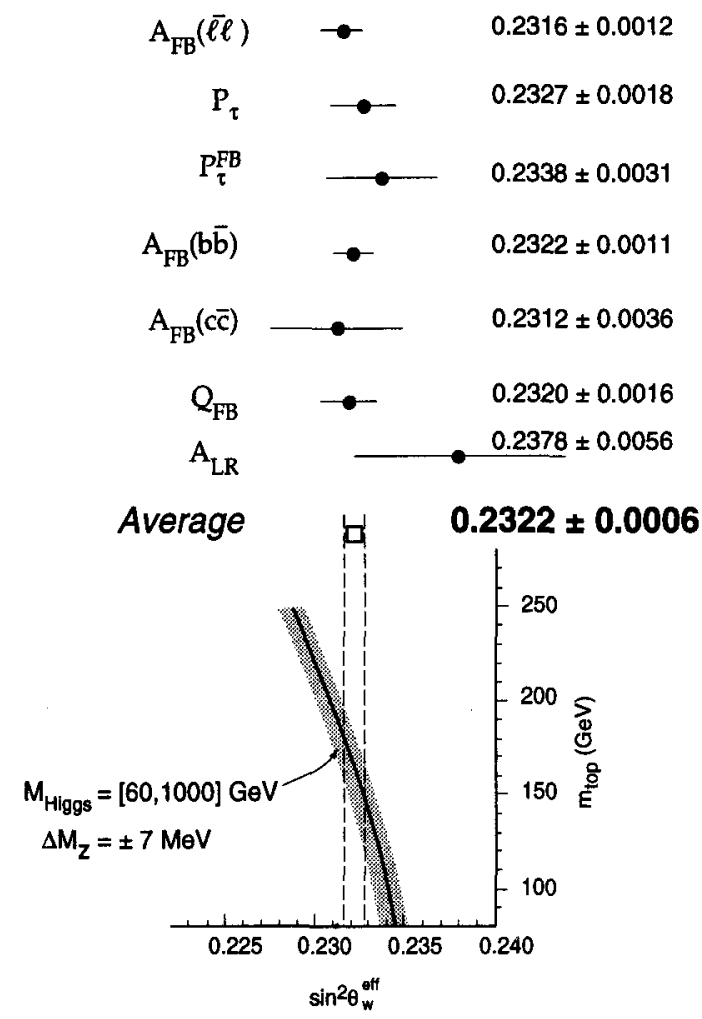

Figure 5: Various measurements of the effective Standard Model parameter $\sin ^{2}(\theta) W^{\text {eff }}$ at LEP and comparison with Standard Model predictions as a function of the top quark mass and for a broad range of Higgs particle masses. 


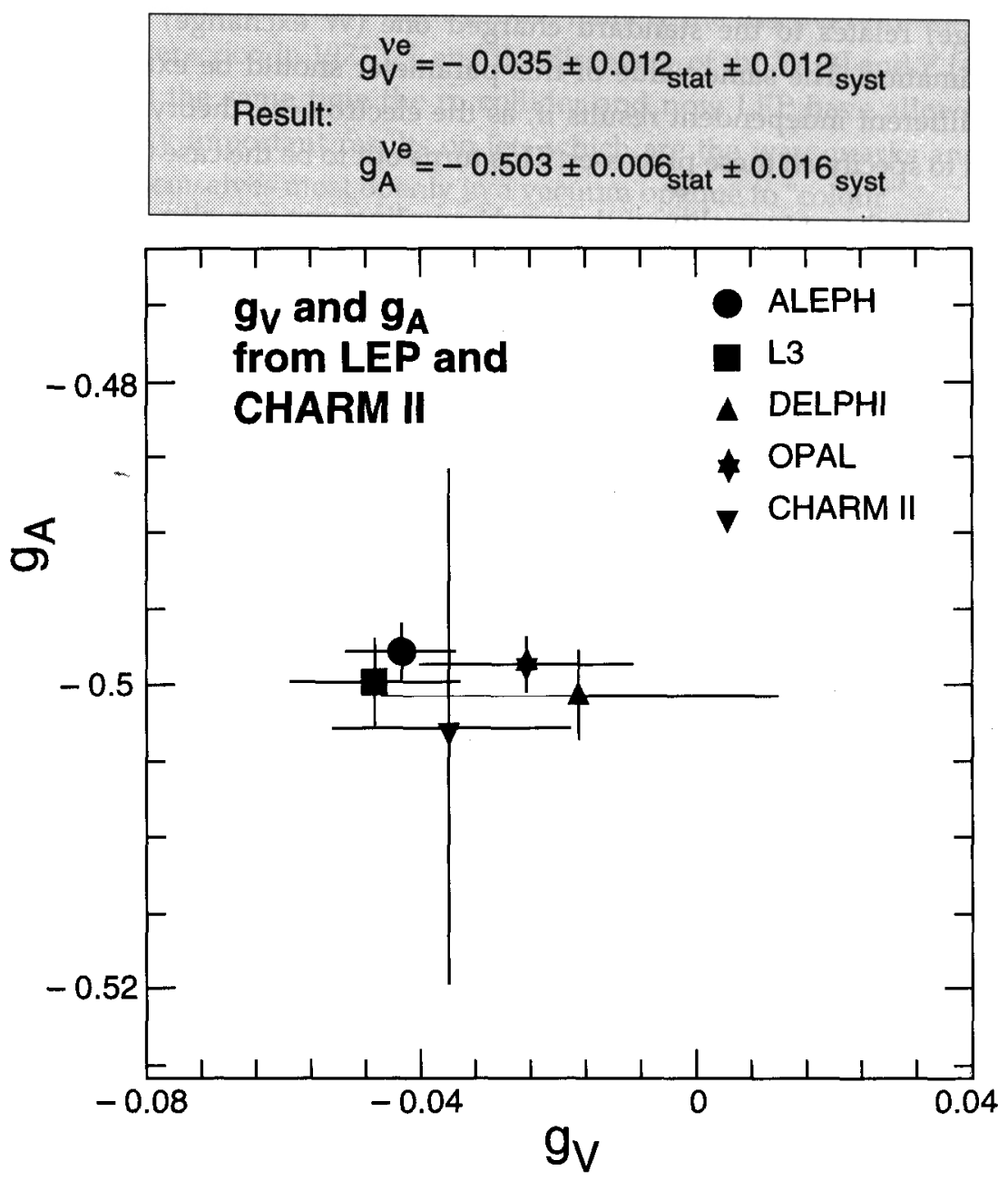

Figure 6: Comparison of LEP (electron-positron scattering) and CHARM-II (neutrinoelectron scattering) experimental measurements of the electroweak vector and axial-vector couplings gV and $\mathrm{gA}$.

Turning now to strong interactions, we can for instance look at the beautiful jet events observed at LEP. The simplest process consists of the formation, in an electron-positron collision, of a $\mathrm{Z}$ which annihilates into a quark-antiquark pair (Figure 7). The quarks carry "colour" and cannot penetrate the vacuum. Their energy and momentum turn into that of jets of hadrons (mainly $\pi$ mesons) which leave little doubt as to their origin. Part of the time the production of the quark-antiquark pair is accompanied by the radiation of an energetic gluon. 
This gives a third jet (Figure 8). Quarks and gluons are thus almost directly "seen".

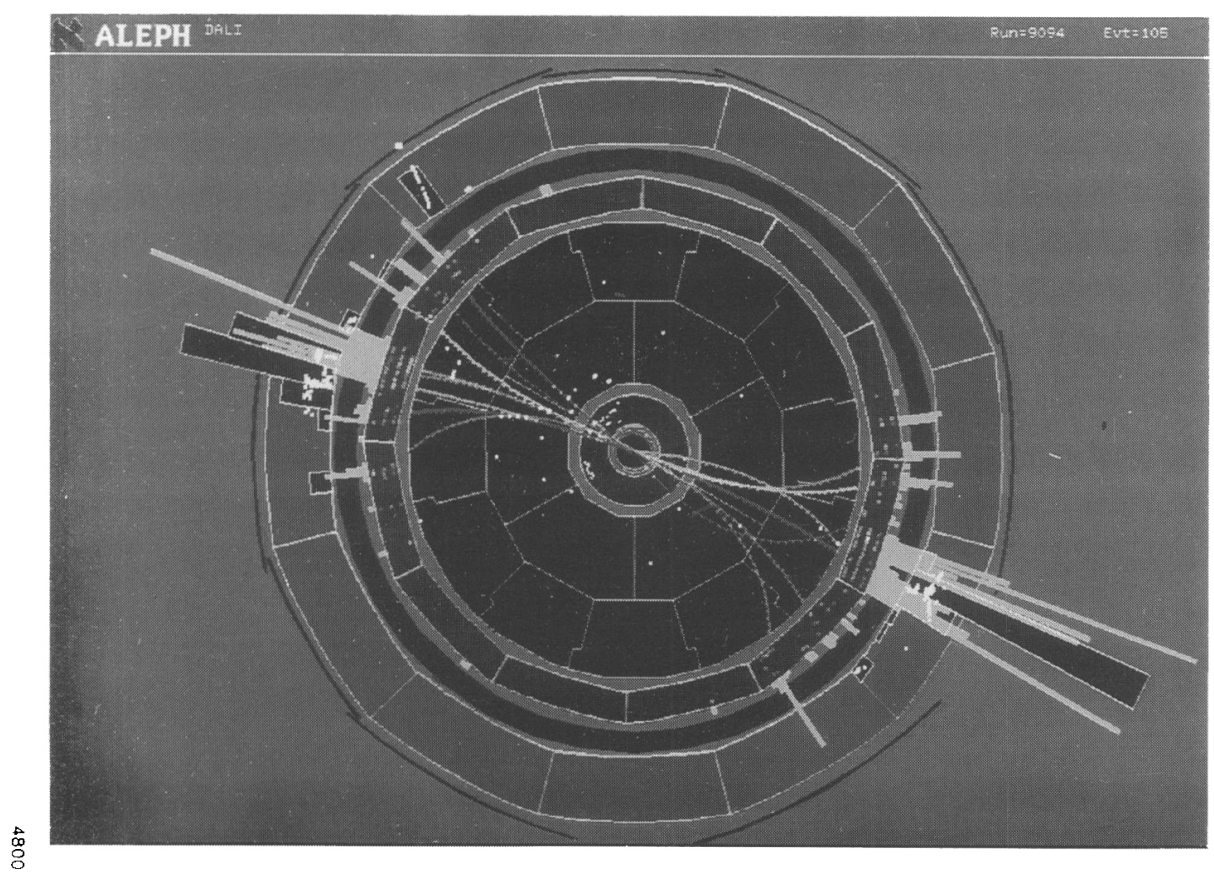

Figure 7: Example of a two-jet-event observed in the ALEPH detector at LEP. The jets of particles are interpreted as the result of a pair quark-antiquark produced in a high energy $e^{+} e^{-}$collision.

One can predict the observed jet pattern in LEP events and many other processes in terms of the unique strong coupling, $\alpha_{\mathbf{S}}$. It "runs" and one can convert all results to a scale corresponding to the $Z$ mass. The same value is found as required (Figure 9). One can now quote a value of $\alpha_{S}\left(M_{Z}\right)$ of 0.118 with an error of 0.006 [9]. Measuring it at different energy scales, one has clear evidence for its "running" (Figure 10), even within the LEP experiments alone, since they obtained data at the tau lepton mass scale [10] in addition to the $Z$ mass scale.

This overview of the Standard Model and of some of its great successes may lead us to expect that particle physics is almost "finished", or rather almost completed. One may be tempted to think that we now have a theory which beautifully describes all the experimental results which we can obtain whilst having predicted most of them in the first place. One may think that all there is 
to do is to increase the precision with which we know the parameters. This has, however, already been heard in physics in the last century and, for the very same reasons, it does not apply to particle physics today! The field is very much alive with new results and burning questions.

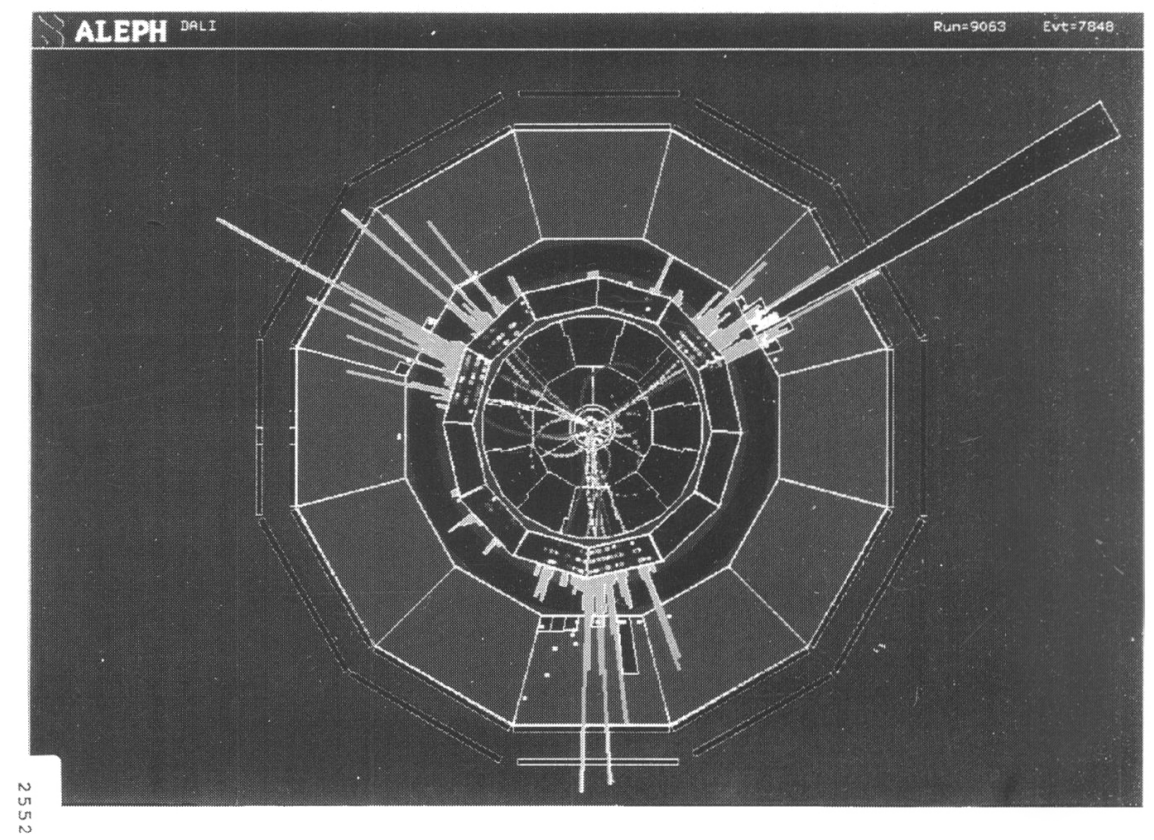

Figure 8: Example of a three-jet-event observed in the ALEPH detector at LEP. The jets of particles are interpreted as the result of a pair quark-antiquark accompanied by a gluon, produced in a high energy $\mathrm{e}^{+} \mathrm{e}^{-}$collision.

\section{The God's Particle}

The Higgs mechanism was invented in the Standard Model to generate masses for quarks, charged leptons and some of the gauge particles. The vacuum is filled by the Higgs field whose fluctuation quanta correspond to the presence of a neutral spin zero particle $\left(\mathrm{H}^{0}\right)$, the Higgs boson. One or several Higgs multiplets, charged and neutral, are possible and indeed likely!

Experimentally, "proving" the validity of the Higgs mechanism is generally interpreted as discovering such scalar particles and verifying their main properties: couplings proportional to fermion masses etc. 


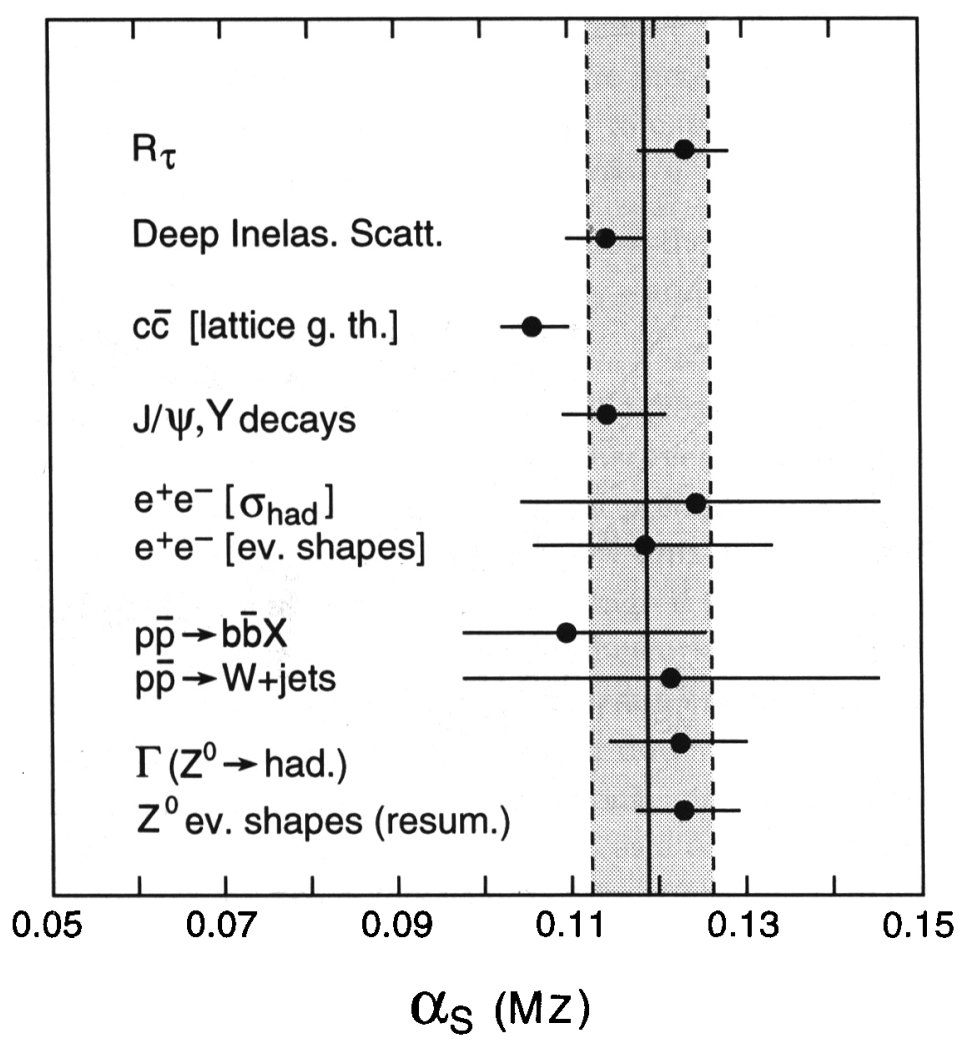

$$
\alpha_{S}\left(M_{Z}\right)=0.118 \pm 0.007
$$

Figure 9: Summary of measurements, at the $Z$ mass scale, of the strong coupling constant $\alpha_{\mathbf{S}}$.

Theoretically, the Higgs mechanism is not without difficulties: the $\mathrm{H}^{0}$ mass is not "protected" and it will automatically "float" to the highest limit of validity of the theory. One solution is to introduce Supersymmetry, i.e. for each known particle there is another one, in a different spin state, which has to be discovered. This is good news for people concerned with (cold) dark matter ! Some of the supersymmetric particles could indeed constitute a large fraction of the dark matter. Note also that the effect of the Higgs field on the evolution of the universe is tremendous because of its large contribution to the cosmological constant. 


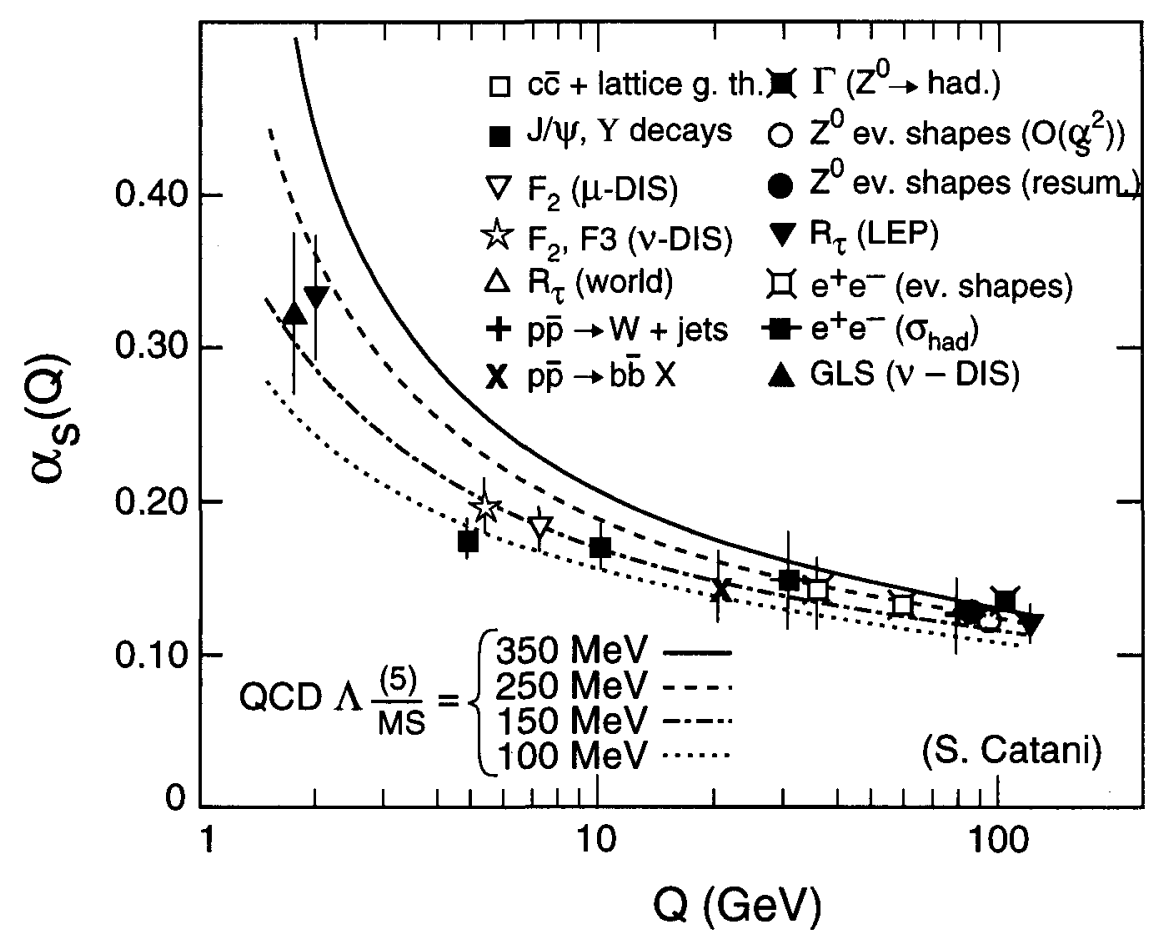

Figure 10: The evolution of $\alpha_{S}$, the strong coupling constant, as a function of momentum transfer $Q$, showing that it is indeed running.

As repeatedly emphasised, understanding the deep nature of symmetry breaking in the Standard Model is a key question in particle physics. The Higgs may be found at LEP-200 but for that its mass should be less than $90 \mathrm{GeV}$ [11]. The production cross-section would otherwise be too small. The Higgs could be more massive and its observation would then require a much higher collision energy.

We would however not like to have it too massive since the applicability of perturbation theory, which is a great asset of the Standard Model, would then break down. For instance if the Higgs mass is $500 \mathrm{GeV}$, perturbation theory would already break down at $1 \mathrm{TeV}$. The lower the mass the further perturbation theory can go. Let us, however, keep an open mind. If perturbation theory no longer holds at high energy, there would be strong interaction effects seen in WWor $\mathrm{ZZ}$ scatteringas we reach beyond $1 \mathrm{TeV}$. They would be within the range covered by the LHC.

Talking about a very heavy Higgs, one is greatly worried that, in the framework of a GUT, nothing a priori prevents the Higgs mass from being 
driven up to the GUT energy scale through radiative corrections. Keeping the Higgs mass at a reasonable value would require incredibly fine tuning in the parameters of the theory. One is therefore led to expect something far more drastic and this motivates our urge to go and look.

There are three main lines of thought. Let us follow the first one - it is the more traditional one in which the Higgs is elementary - and search for it over a very wide mass range.

To that effect, one can consider how any specific cross-sections vary with the machine energy (Figure 11) and assess the discovery potential for a given luminosity. One can get important hints from precision measurements but, most of the time, there is nothing like energy ! This certainly applies to the Higgs. As we said, the mass should not be too high if the Standard Model is to keep its perturbative property. Within the framework of the Standard Model, the Higgs production mechanism and cross-section are entirely determined if its mass is known (Figure 12), and there is little doubt that if it exists it will be discovered. Using the present measurements of the Standard Model parameters, one can constrain the Higgs mass as a function of the top quark mass [49] (Figure 13). Relatively low values of the Higgs mass are favoured.

However, we have also Grand Unification in mind and we cannot help keeping wondering how the Higgs mass could be kept low. This is where Supersymmetry (SUSY) comes into the picture in the most dramatic way. Supersymmetry is a symmetry between fermions and bosons, mixing geometrical and internal degrees of freedom. It has been with us for two decades already. It is very appealing theoretically but the main problem with it is that "sparticles", the supersymmetric partners of the existing particles, have been extensively looked for and not found so far [12]. The only thing which we can say from the LEP results is that their masses are all in excess of $50 \mathrm{GeV}$. However, this could well be the case if they exist, since supersymmetry is broken in the real world and "sparticles" could be very massive. One should therefore keep trying with increasing energy.

The presence of supersymmetric partners to the existing particles is very welcome in the framework of our Higgs mass problem since radiative effects 


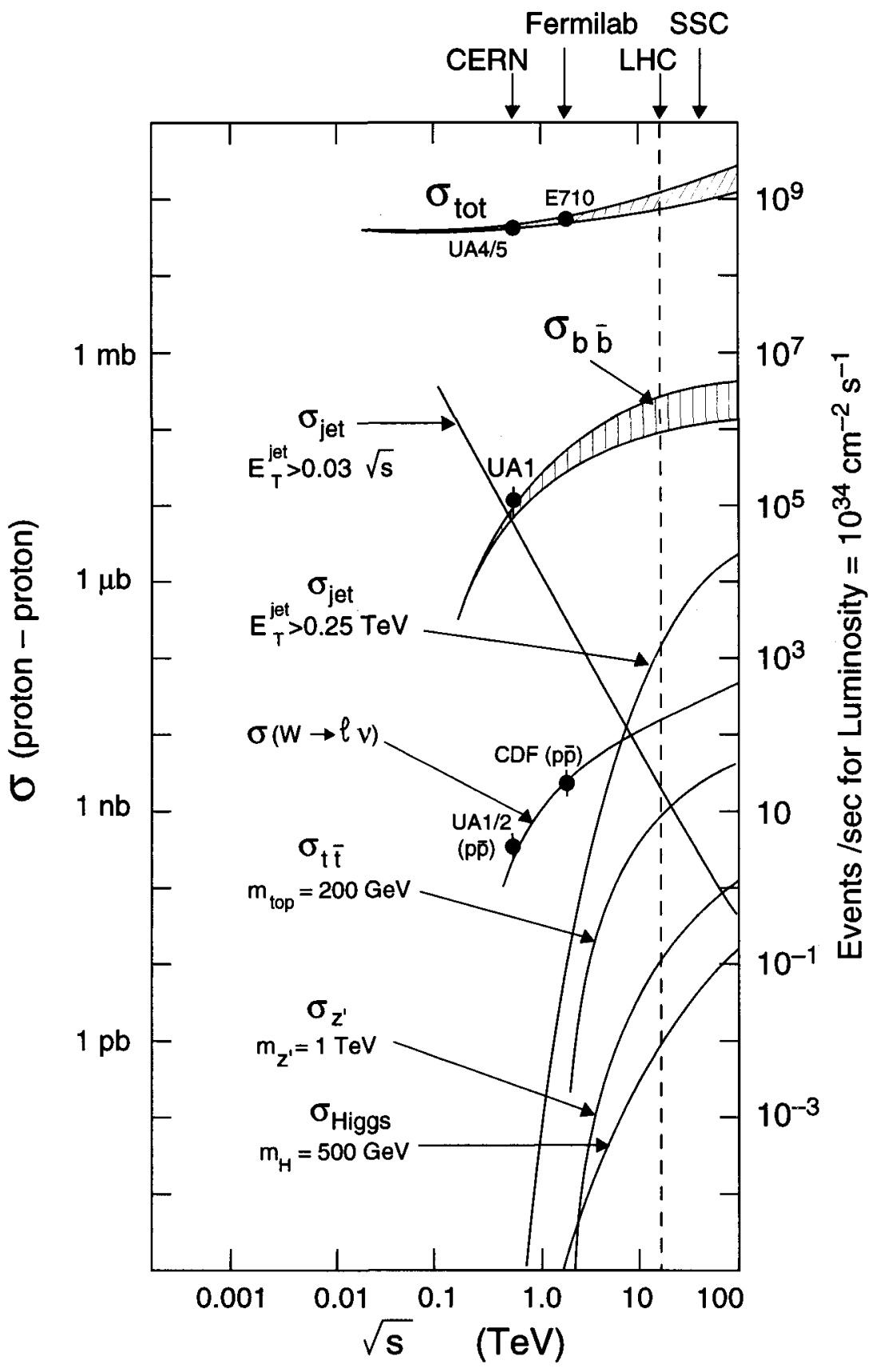

Figure 11: Cross-sections for various reactions in proton-proton collisions as a function of centre of mass energy. 
$\mathrm{H}^{\circ}$ production at hadron colliders:
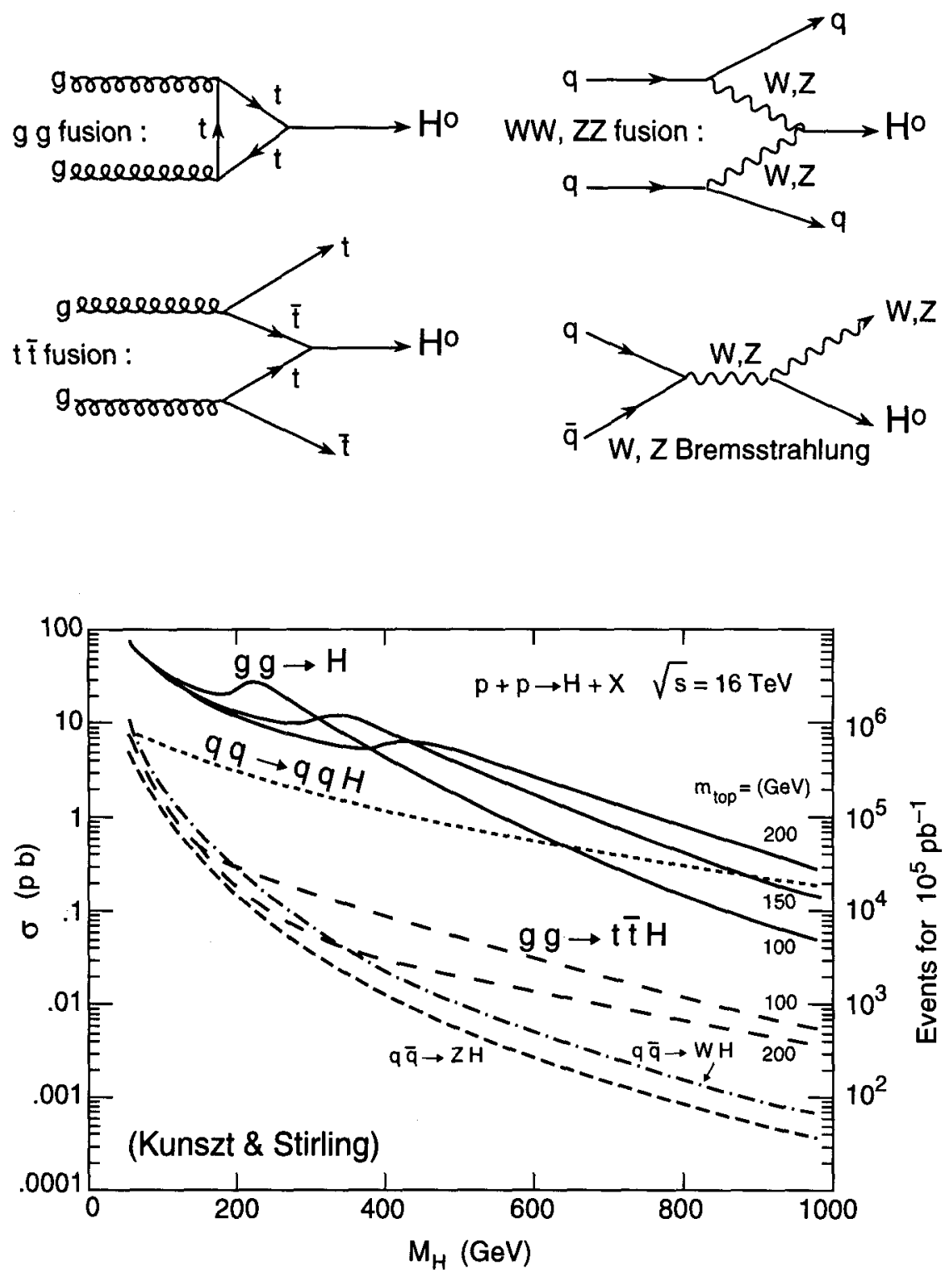

Figure 12: The production cross-section for the Standard Model Higgs and for the main subprocesses contributing in proton-proton collisions. The corresponding Feynman graphs are also shown. 
would then globally cancel themselves out. Not fully though and, if one wishes to keep the Higgs mass low, the supersymmetric partners could not be too massive. We are therefore led to focus on a mass range extending at most from a few $\mathrm{GeV}$ to several hundreds of $\mathrm{GeV}$. This is very nice because, if this were the case, the LHC would then have a great discovery potential.

If "sparticles" exist, the running of the coupling constants is modified at energies beyond their production threshold. The precise LEP results indeed hint that this could be the case [5]. This would be the way to reach a common GUT value assuming that there is nothing drastic happening for energies in between.

The lightest supersymmetric particle should be stable and very weakly interacting because its interactions require the exchange of a very massive supersymmetric particle. It could then be an interesting candidate for cold dark matter [13].

Supersymmetric particles are of course a consequence of superstring theories but nothing can then be said about their masses which could perhaps be very heavy. On the other hand, observing "sparticles" would be a strong experimental boost for superstring theories which are still "stuck" at the Planck scale.

For all these reasons SUSY appears as an extremely interesting approach. There are many ways to introduce symmetry breaking in a SUSY theory but one is tempted to make "sparticles" play an important role at not too high an energy. One attaches, therefore, some particular interest in a Minimal Supersymmetric Standard Model, with a minimal number of extra parameters on which one can already put some constraints. The supersymmetric partners have their mass upper bounds lower than $1 \mathrm{TeV}$, and lower than $200 \mathrm{GeV}$ for one of them! This, therefore, also puts them within the discovery range of the LHC. There are five Higgs particles in the model, including three neutrals. This is then a strong encouragement in our search for the lightest Higgs, as a first wanted particle on the list.

Another line of approach is more drastic and it does not make use of the appealing theoretical framework provided by supersymmetry. One assumes that the complications with a very high Higgs mass are actually not very relevant since the Higgs is a composite particle after all. It could be a bound state of hitherto unknown fermions and there are theoretical models along that line. The most popular one follows QCD calling for a "technicolour" interaction 
involving hitherto unknown massive "techni-particles". These models have had ups and downs in coping with existing data but they have remained alive for many years. If this is the way Nature works, thresholds could not be too high since the main goal is after all to keep the Higgs mass low. There is, therefore, a full new zoo of techni-particles which we could discover in the energy range offered by the LHC. This would reveal a further new layer in the structure of matter.

The third line of approach is to keep a far more open mind and be ready for something even newer and a priori more exotic. Again the energy range is appropriate for such surprises. To illustrate this, one may say that the successes met within gauge theories lead us to think that all symmetries should be gauge symmetries unless they are accidental. One is then led to challenge even baryon and lepton conservation and could think of baryon number and lepton number violation already at the electroweak scale. This is the "sphaleron" approach. If the temperature can exceed the electroweak critical temperature in a very high energy collision, hosts of particles could be produced with violation of our standard conservation laws. In the early universe this would lead to an erasure of the primordial production of quarks and leptons at the end of the GUT era. This is rather radical but we should be ready for surprises since we are seeking to understand a fundamental symmetry breaking mechanism through which particles acquire their masses.

Whatever line of thought is followed, one can say that probing physics in the $\mathrm{TeV}$ to a few $\mathrm{TeV}$ range and thus reaching an understanding of the structure of matter down to $10^{-19} \sim 10^{-20}$ metre, is entering an a priori very rich domain.

\section{Beyond the Standard Model: the need of a new phenomenology at unreachable energies}

Naively, the structural similarities between the electroweak theory $[S U(2) \times U(1)]$ and $Q C D[S U(3)]$ together with the fact that their coupling constants seem to approach one another with increasing energy to eventually meet around $10^{16} \mathrm{GeV}$, strongly suggests a GUT where quarks and leptons would appear as different manifestations of the same phenomenology. This implies proton decay, a process which we should be very much concerned with. 


\subsection{Status of matter stability}

There is no known reason to believe that baryon number conservation is exact - unlike, for instance, the conservation of electric charge which is directly linked to gauge invariance and to the existence of long range interactions. Eötvös-type experiments found no evidence for the existence of a long-range field coupled to the baryon number down to a level of $10^{-9}$ times the gravitational force [14].

The universe is a gigantic example of baryon number violation! To explain the observed predominance of matter over antimatter and the current ratio between photons and baryons $\left(n_{\gamma} / n_{B} \sim 10^{10}\right)$, cosmological theories must assume that, in the very hot initial stages of the Big Bang, there were frequent baryon non-conserving interactions coupled at some level to $\mathrm{CP}$ violating processes. Those interactions must lead to proton decay. The main question is: at which level? If the relevant scale is the Planck mass, then the proton lifetime is of the order of $10^{44}$ years, and detecting it would be a hopeless experimental task. Statistically it would take at least $10^{10}$ Superkamiokande's, but even that would not work because of the overwhelming background.

However, most GUTs, in which baryon non-conservation occurs naturally, require some intermediate mass scale, determined by the mass of the Grand Unification bosons. With LEP precision measurements of $\sin ^{2} \theta_{W}$ and $\alpha_{S}\left(M_{z}\right)$, the extrapolation of the gauge coupling constants to higher energy scales shows that they meet at a point when evolved within the context of a minimal

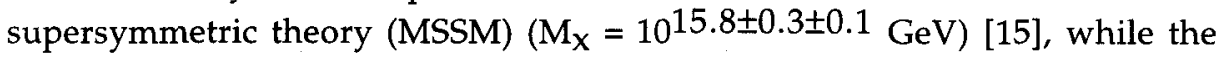
couplings fail to unify within the Standard Model [16], which rules out the simplest one-step GUTs, such as desert-scenario $\mathrm{SU}(5), \mathrm{SO}(10)$, etc. Such a colossal energy is still far above that of any known elementary process occurring at present and will probably never be reached by means of accelerator techniques. However, it must have been reached in the very early stages of the life of our universe $\left(10^{-40}\right.$ to $10^{-35} \mathrm{~s}$ after the Big Bang). Therefore, GUTs are naturally relevant to the creation and decay of matter. Proton decay, if detectable, represents a direct way to investigate physics at the Grand Unification scale, and could play a role analogous to $\beta$-decay which taught us almost everything about the Standard Model.

Early proton decay searches, in the mid 1970's, were stimulated by the first and simplest GUT, based on SU(5). The "desert-scenario" non-supersymmetric 
$\mathrm{SU}(5)$ GUT predicts a value of $\mathrm{M}_{\mathrm{X}}$ at $\mathrm{O}\left(10^{14}\right) \mathrm{GeV}$, which, via the dominant $\mathrm{p} \rightarrow \mathrm{e}^{+} \pi^{0}$ decay mode, yields a proton lifetime:

$$
\tau_{p} \sim \frac{M_{X}^{4}}{\alpha_{G}^{2} m_{p}^{5}} \sim 10^{31 \pm 1} \text { years }
$$

a value somewhat below the current experimental limit $\tau_{p}>5.5 \times 10^{32}$ years. With the SU(5) GUT in trouble, proton decay experiments were no longer considered fashionable; however, great progress had been accomplished with the pioneering experiments, bringing the proton lifetime limits from $10^{29}$ to $10^{32}$ years!

Unfortunately, in globally super-symmetric GUTs, the proton lifetime which depends not only on the GUT physics but also on the low energy superparticle spectrum cannot yet be quantified (137 free parameters) and lies generally in a wide range of values: $\tau_{p} \sim 10^{33}$ to $10^{38}$ years. Operators exist (so-called dimension-5) leading to proton decays which must change generation. Such channels are characterized by the presence of strange particles in the final state, with the following selection rule:

$$
\frac{\Delta \mathrm{S}}{\Delta \mathrm{B}}=0,-1
$$

which tells us that $\mathrm{p} \rightarrow \overline{\mathrm{v}} \pi^{+}$or $\bar{v} \mathrm{~K}^{+}$are allowed, but that $\mathrm{p} \rightarrow \overline{\mathrm{v}} \mathrm{K}^{-} \pi^{+} \pi^{+}$or $\mathrm{n}$ $\rightarrow \mathrm{e}^{+} \mathrm{K}^{-}$are forbidden.

The situation is remarkably different in the supergravity GUT [17] (SUGRAGUT) which generates its own spontaneous breaking of supersymmetry. The soft supersymmetry breaking terms in the low energy theory are completely determined in terms of only four parameters. Such a theory will definitely be tested by a combination of LEP and future proton decay experiments, since it predicts that either $\tau\left(p \rightarrow \bar{v} K^{+}\right)<1.5 \times 10^{33}$ years or one of the gauginos or one of the Higgs particles will be discovered at LEP200 [18]. This is a really exciting challenge both for proton decay and LEP200 experiments.

The discovery of proton decay in the supersymmetric channel would obviously be of great importance. However, from the experimental point of view, it is clear that all the possible decay modes should be searched for, without too much theoretical prejudice. In addition, the present experimental situation has 
room for substantial improvement, even for decay modes with $\tau / \mathrm{Br} .<10^{32}$ years. For instance, for the apparent anomaly first observed by Kamiokande in atmospheric neutrino data [19], the possibility that the source of the effect is in fact proton decay has been seriously considered [20].

Present Limit $\square \quad \square$ Superkamiokande (5 years)

- ICARUS (10 year.module)

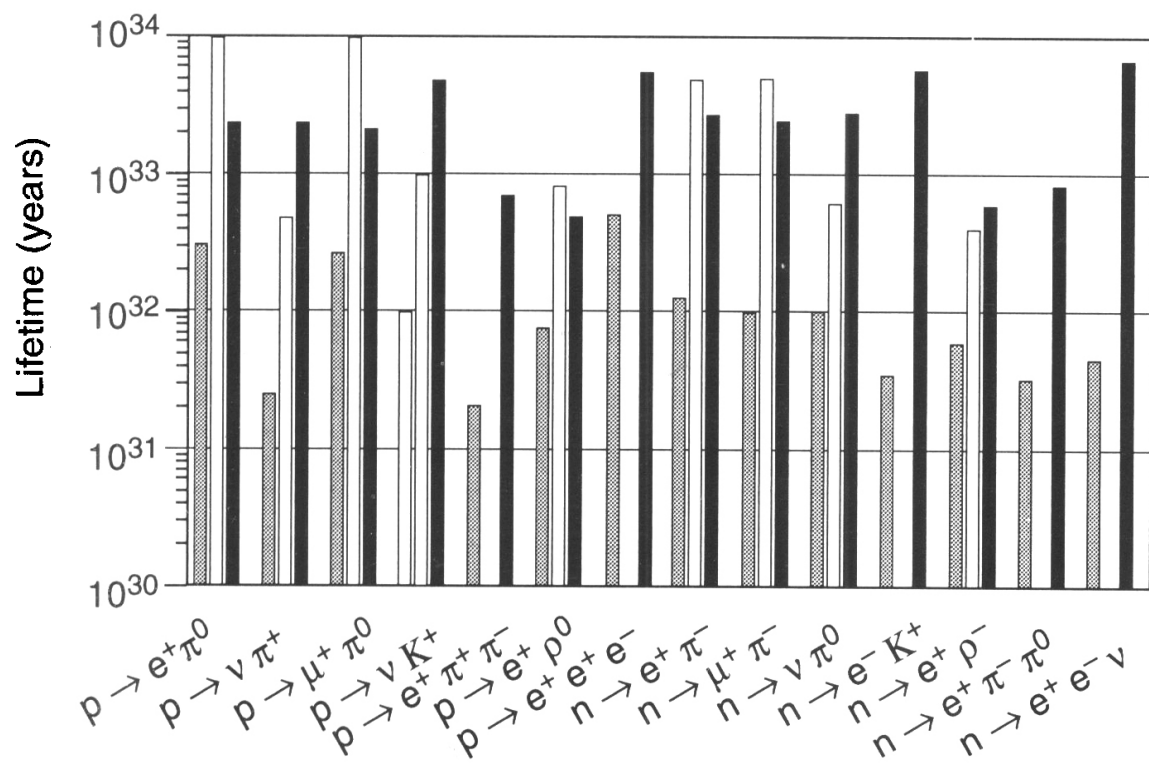

Figure 14: Sensitivity for Superkamiokande and ICARUS and for present proton and neutron decay limits in a selected number of channels.

The current generation of detectors has reached the proton lifetime range of $10^{32}$ years (Figure 14), but only for a limited number of channels. There is the possibility that the current Soudan II detector [21], with a sensitive mass of 1 Kton, may still explore some additional channels, in that range. In order to go further and investigate the region $\tau_{\mathrm{p}} \sim 10^{32}$ to $10^{34}$ years which seems to be indicated by SUGRAGUT, two approaches are presently considered: (a) increase the mass of the sensitive medium using current detector technologies (the Superkamiokande approach, factor 30). Due to the relatively large atmospheric neutrino background, the signal-over-noise ratio is small and the sensitivity is proportional to $\mathrm{VN}_{\mathrm{n}}$, ( $\mathrm{N}_{\mathrm{n}}$ is the number of nucleons); (b) use new detector technologies to obtain a negligible background over a more modest 
detector size (the ICARUS approach). The signal-over-noise ratio is large and the sensitivity is proportional to $\mathrm{N}_{\mathrm{n}}$.

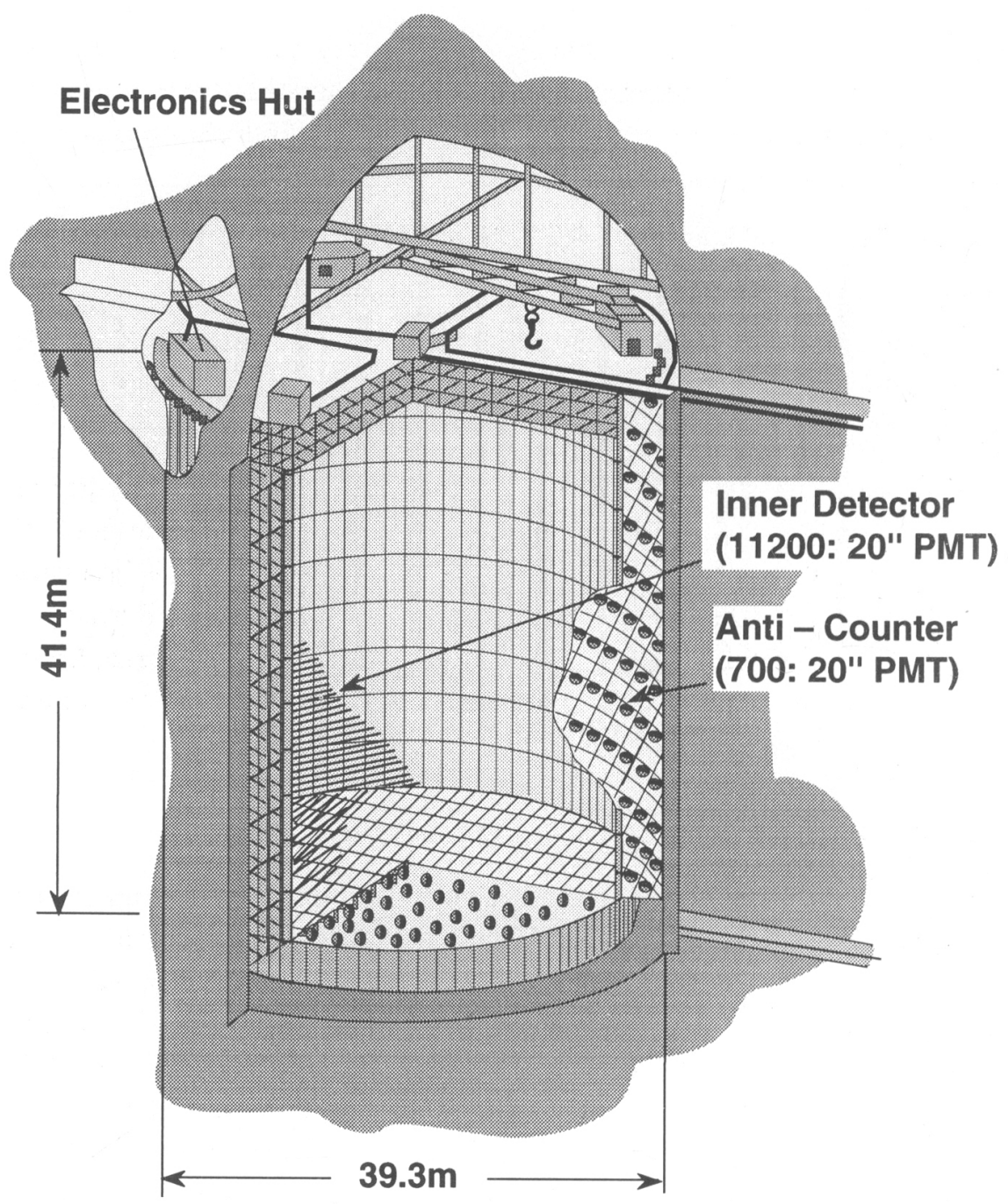

Figure 15: Schematic view of the SuperKamiokande detector.

\subsection{Superkamiokande}

Superkamiokande [22] (Figure 15) is a spectacular extension of the Kamiokande technique (water Cerenkov) to a much larger fiducial mass: 22,000 tons with a significant improvement in low energy event reconstruction mainly 
due to a better photomultiplier tube coverage, $40 \%$ versus $20 \%$. The detector is built in the same Mozumi Zinc and Lead Mine as the present Kamiokande experiment in Japan (200 m west-southwest of Kamiokande).

Excavations which began in November 1991 are now completed. Data taking is expected to start in 1996. Superkamiokande will, in five years, reach proton or neutron decay lifetimes of up to $10^{34}$ years for certain channels such as $\mathrm{p} \rightarrow$ $\mathrm{e}^{+} \pi^{0}$ and $10^{33}$ years for $\mathrm{p} \rightarrow \overline{\mathrm{v}} \mathrm{K}^{+}$(Figure 14 ).

\subsection{Imaging Cosmic And Rare Underground Signals (ICARUS)}

ICARUS [23] (Figure 16) at the Gran Sasso underground laboratory in Italy, is a large (three 5,000-ton modules) liquid argon TPC. It is an "electronic" bubble chamber with self-triggering capability and with many precise $\mathrm{dE} / \mathrm{dx}$ samplings along particle paths.

A 3-ton prototype is presently operating successfully at CERN [24] and has demonstrated excellent space and energy resolution and particle identification. The redundancy of information allows negligible backgrounds and discovery at the single event level, corresponding to lifetimes of up to $10^{34}$ years, in channels such as $\mathrm{p} \rightarrow \overline{\mathrm{v}} \mathrm{K}^{+}$(Figure 17) or $\mathrm{n} \rightarrow \overline{\mathrm{v}} \mathrm{K}^{0}$. The unique strength of ICARUS is the possibility of studying many exclusive nucleon decay channels. The first detector module is expected to be completed in 1998.

\section{Beyond the Standard Model: neutrino masses and mixing}

Proton decay and neutrino oscillations have in common that they may both allow the study of physics at the GUT scale and, in addition, they are often searched for in the same detector. Twenty years of solar neutrino experiments pioneered by $R$. Davis have promoted neutrino physics to a new frontier comparable in importance to the high energy frontier (SLC, LEP, TEVATRON and $\mathrm{LHC}$ ) and the high precision frontier (LEP, $\Phi, \mathrm{B}, \mathrm{K}, \tau$-charm factories). Yet, today, neutrinos are still poorly known and our main question is simple: "Do they have a mass?". In the Standard Model neutrinos are massless but without compelling reason (arbitrariness). Theories beyond the Standard Model which may explain some of its arbitrary features lead us to believe that neutrinos should be massive. For instance, "see-saw" models [25] provide a natural extension of the Standard Model, in which neutrino masses originate from couplings with right-handed Majorana partners, too heavy to be observed. 


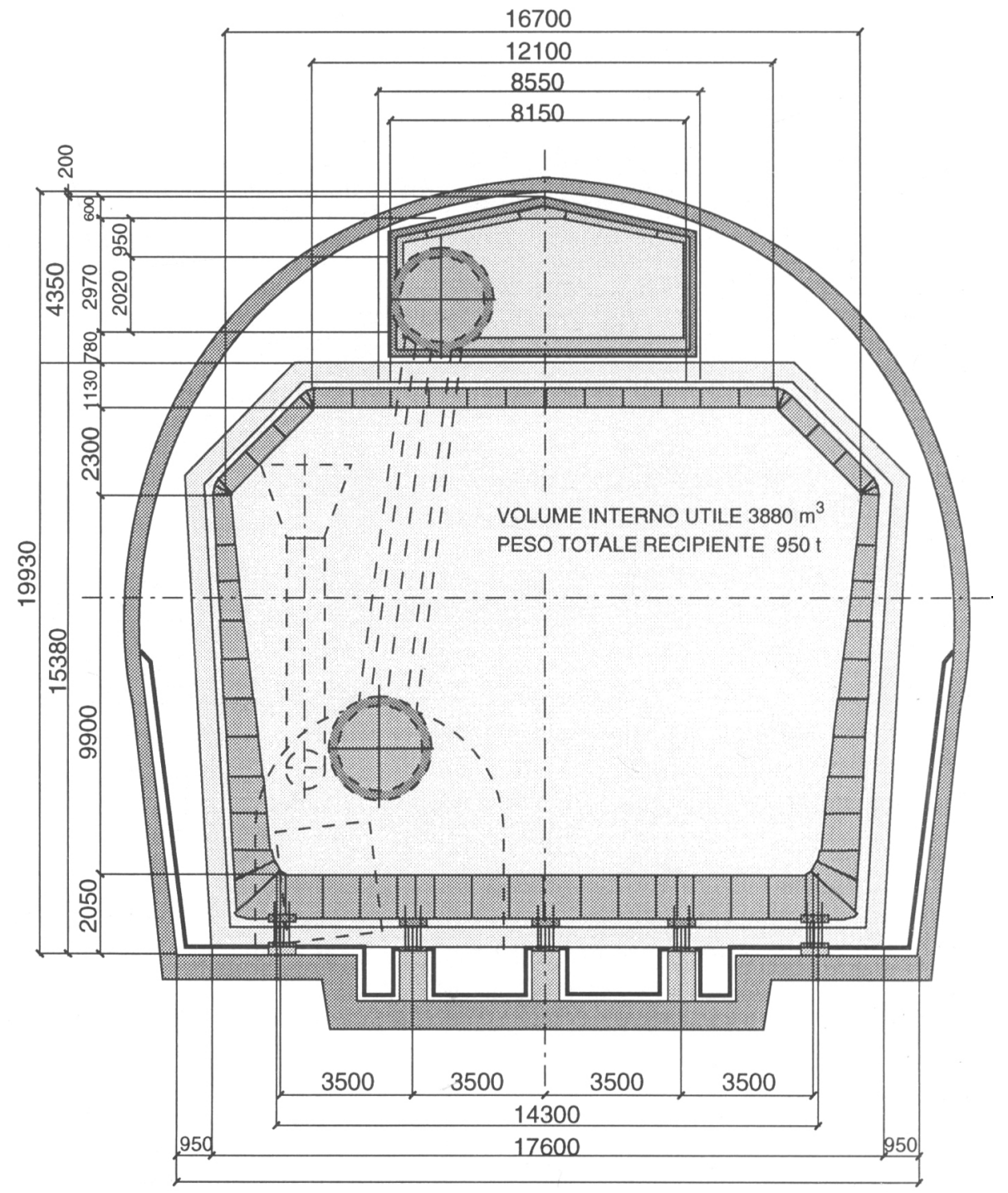

Figure 16: Schematic view of the ICARUS detector to be built in the Gran Sasso Laboratory. 


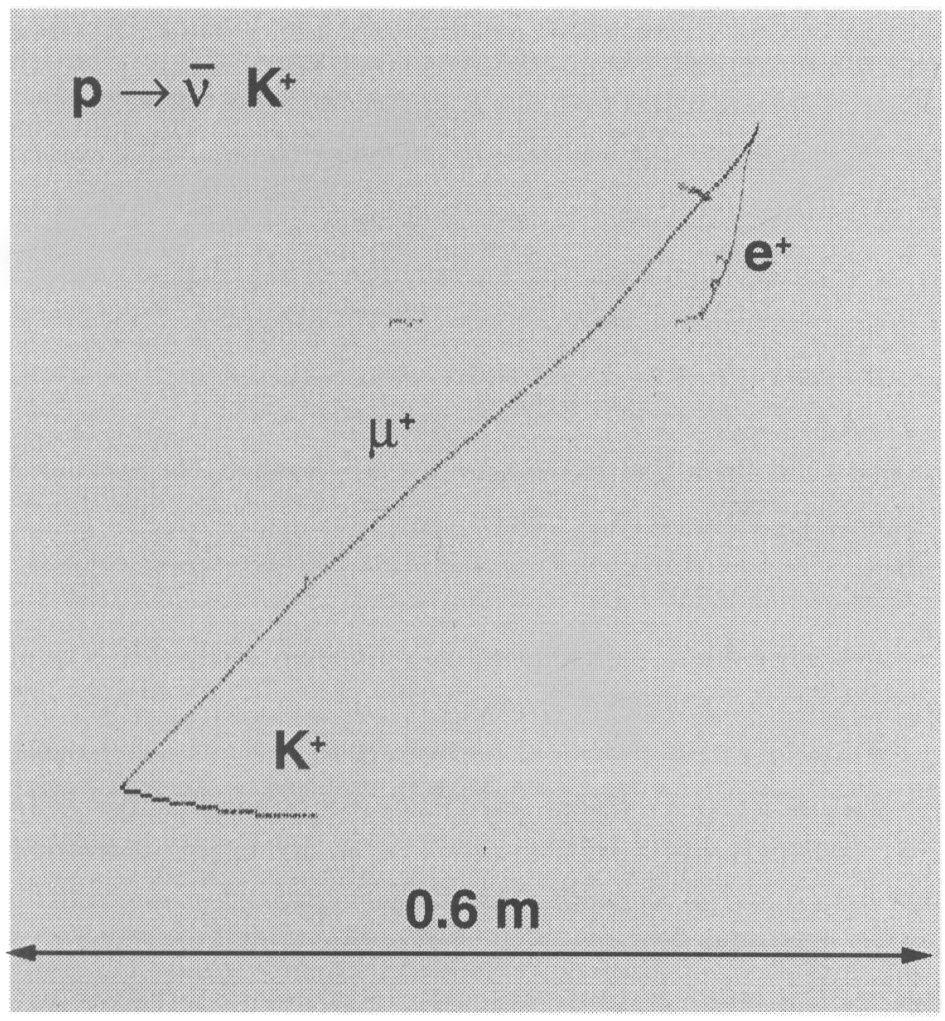

Figure 17Simulation in the ICARUS detector of a proton decay in the Supersymmetrically preferred mode $\mathrm{p} \rightarrow \overline{\mathrm{v}} \mathrm{K}^{+}$.

In these models and for three flavours, one is generally led to three light lefthanded Majorana neutrinos (the observed $v_{e}, v_{\mu}, v_{\tau}$ ) with mass matrix $m_{v}=$ $\mathrm{mDMN}^{-1} \mathrm{mD}^{\mathrm{T}}$ (each entry in the previous expression is a $3 \times 3$ matrix) and three superheavy right-handed Majorana neutrinos, corresponding to unobserved massive particles. Both quarks and leptons are expected to have masses of order mD. We can distinguish two simplifying cases: (a) $M_{N}$ is proportional to the identity matrix resulting in the quadratic see-saw: $m_{v}=$ $\mathrm{mD}^{2} / \mathrm{MN}_{\mathrm{N}}$; (b) $\mathrm{MN}_{\mathrm{N}}$ is proportional to $\mathrm{mD}_{\mathrm{D}}$ resulting in the linear see-saw: $\mathrm{m}_{\mathrm{V}}=$ $\mathrm{mD} / \mathrm{MN}$.

To some extent, we may say that we do understand the smallness of neutrino masses from the fact that the $\mathrm{MN}_{\mathrm{N}}$ terms are very large. However, in general the question remains of knowing whether we should use a linear or a quadratic see-saw, with quarks or with leptons. Considering ratios of neutrino masses 
eliminates the overall unknown mass scale $\mathrm{M}_{\mathrm{N}}(\mathrm{X})$. Assuming, for instance, that we know the muon neutrino mass, we can compute the tau neutrino mass, modulo radiative corrections! Unfortunately, the answer for $m_{v \tau}$ still varies by three orders of magnitudes! A more definite theory is needed to provide clear predictions. Most predictive are GUTs which naturally give $\mathrm{m}_{\mathrm{D}} \propto \mathrm{m}_{\mathrm{u}}$ quarks.

Conversely, within these models, the knowledge of $m_{v}$ implies the knowledge of the $\mathrm{M}_{\mathrm{N}}$ mass scale. For instance, in the SO(10) minimal SUSY GUT [25], the LEP precision data on the interaction couplings, are consistent with a unification scale $\mathrm{MX}=1.6 \times 10^{16 \pm 0.4} \mathrm{GeV}$ and a SUSY symmetry breaking scale $\leq 1 \mathrm{TeV}$. Perhaps the main feature to retain from these GUTs is a definite mass hierarchy: $\mathrm{m}_{v e}: \mathrm{m}_{\mathrm{v} \mu}: \mathrm{m}_{\mathrm{v} \tau}=\mathrm{m}_{\mathrm{u}}^{2}: \mathrm{m}_{\mathrm{c}}^{2}: \mathrm{m}_{\mathrm{t}}^{2}$.

\subsection{Present solar neutrino experiments}

The solar neutrino problem stems from four experiments, still actively taking data: Kamiokande using water Cerenkov techniques, and Homestake, SAGE and GALLEX using radio-chemical techniques. The results from GALLEX $(79 \pm 10-5$ SNUf confirm a certain deficit compared to the predictions of J. Bahcall (132 $-17 \mathrm{SNU})$ and Turck-Chièze (123 $\pm 7 \mathrm{SNU})$. It is not established whether this deficit is due to experimental errors, a poor understanding of the sun or to new neutrino physics. It is therefore crucial to obtain additional information. A first step in that direction is an absolute calibration of the present detectors. In this respect, the GALLEX collaboration is performing such a test with a powerful $(1.7 \mathrm{MCi})$ radioactive ${ }^{51} \mathrm{Cr}$ source. As we write this text we have just heard from GALLEX that the ratio of observed events to expected events they detect from their radioactive source is $1.02 \pm 0.14$ [26]. This important result strengthens GALLEX conclusions on pp neutrinos from the sun.

The most popular explanation for the apparent solar neutrino deficit, if it is to be attributed to new physics, is the Mikheyev-Smirnov-Wolfenstein (MSW) mechanism [27]. However, there may not be a solar neutrino deficit [28], in case flaws exist in present solar models, i.e. several effects could decrease $T_{C}$; plasma effects could affect the $\mathrm{p}+{ }^{7} \mathrm{Be} \rightarrow{ }^{8} \mathrm{~B}+\gamma$ rate, the cross-sections for this process may not be known accurately enough in the range 1 to $10 \mathrm{keV}$ relevant in the sun; diffusion effects; etc. Recently, a solar model consistent with some of the experimental results, was proposed [29] but it is the object of fierce controversy between expert theoreticians [30]. It is clear that the ball is more than ever in the experimentalist's camp! 
It is unlikely that existing experiments will settle the issue, since none of them individually can distinguish between effects coming from the nature of neutrinos and those due to the neutrino production mechanism inside the sun. Therefore one will have to wait for the next generation of solar neutrino experiments.

\subsection{Future solar neutrino experiments}

\subsubsection{Superkamiokande}

With a solar neutrino rate of 23 elastic events $\left(v_{x}+e^{-} \rightarrow v_{x}+e^{-}\right)$per day, almost two orders of magnitude larger than Kamiokande, Superkamiokande will carry the first high statistics real time solar neutrino measurement.

The statistical precision is sufficient to detect the solar neutrino flux modulation of $7 \%$ due to the eccentricity (0.0167) of the earth's orbit. The experiment should be sensitive to rather small time variations and easily clarify issues such as possible correlation with sun spots etc. However, since Superkamiokande is only sensitive to ${ }^{8} \mathrm{~B}$ neutrinos scattering on electrons and does not measure their energy it is not expected that they can separate out solar model effects.

\subsubsection{ICARUS}

Two reactions are used for the direct observation of solar neutrinos from ${ }^{8} \mathrm{~B}$ : (a) elastic $v_{\mathrm{e}}$-electron scattering; (b) $v_{\mathrm{e}}$ absorption $\left(\mathrm{v}_{\mathrm{e}}+{ }^{40} \mathrm{Ar} \rightarrow{ }^{40} \mathrm{~K}^{*}+\mathrm{e}^{-}\right)$, mainly a super-allowed transition followed by ${ }^{40} \mathrm{~K}^{*} \rightarrow{ }^{40} \mathrm{~K}+1$ or $2 \gamma[2 \mathrm{MeV}]$. With a threshold at $5 \mathrm{MeV}$ on the recoil electron energy, the Standard Solar Model predicts about 2700 and 3000 (Fermi transition only) events/year/module for reactions $a$ ) and $b$ ) respectively. The ratio $R$ of the rates for $a$ ) and $b$ ) provides $a$ direct measurement of the fraction of $v_{e}$ 's which eventually converted to $v_{\mu}$ 's or $v_{\tau}$ 's through oscillations, independent of the magnitude of the initial solar neutrino flux, hence of the solar model. With two years of data ICARUS could detect a neutrino oscillation probability of $20 \%$ or larger.

Several other important projects are in preparation: the Sudbury Neutrino Observatory (SNO), a 1000-ton $\mathrm{D}_{2} \mathrm{O}$ Cerenkov detector [31] in Canada; HELLAZ [32], a 6 ton helium TPC at liquid nitrogen temperature proposed for the Gran Sasso Laboratory; BOREXINO a 100-ton liquid scintillator detector [33] also proposed at Gran Sasso.

Note that both Superkamiokande and ICARUS can study the variation of the solar neutrino flux due to the variation of the distance earth-sun. That 


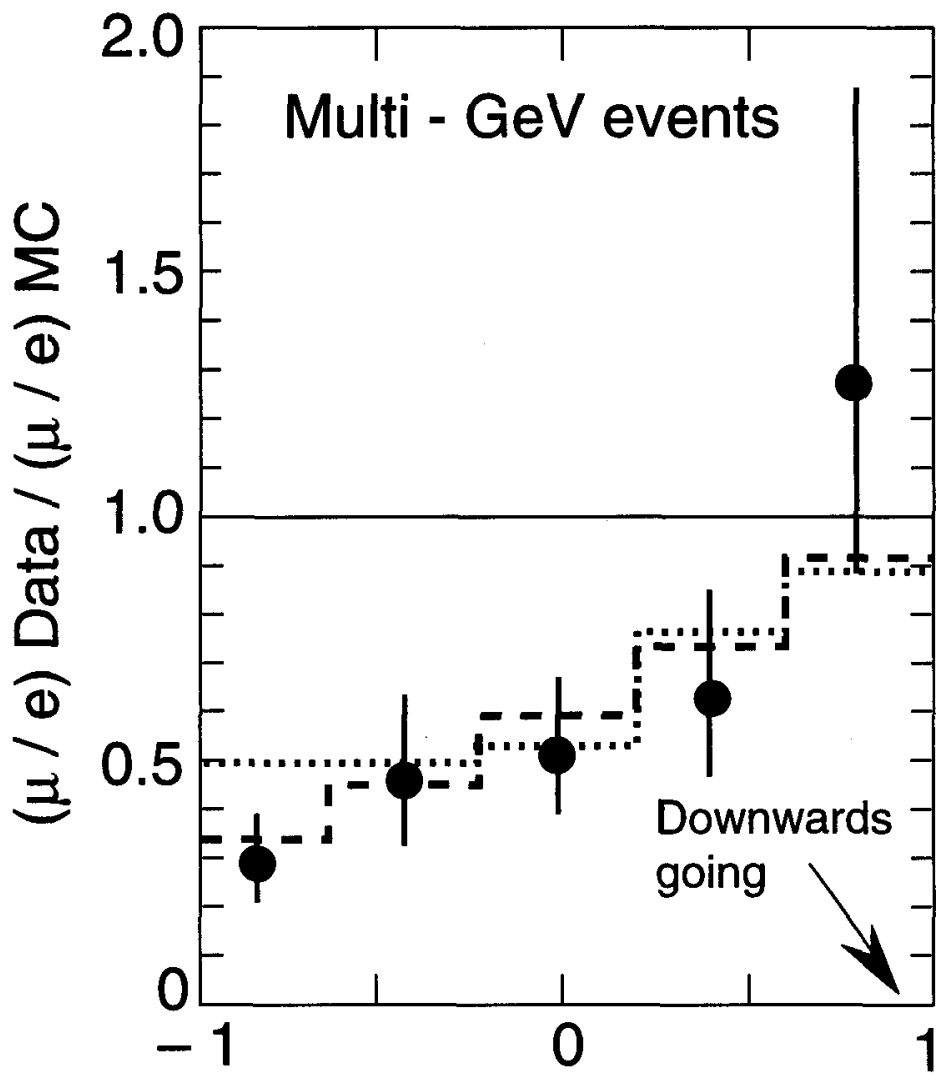

$\operatorname{Cos} \theta$

Figure 19: Kamiokande [35], zenith angle distribution of ( $\mu$-like/e-like) data events over ( $\mu$-like/e-like) Monte Carlo events, for multi-GeV data, where both the fully and partially contained events are included. Also shown are predictions from Monte Carlo simulations with neutrino oscillations with $\Delta \mathrm{m}^{2}=1.8 \times 10^{-2} \mathrm{eV}^{2}$ and $\sin ^{2}(2 \theta)=1.0$ for $v_{\mu} \leftrightarrow v_{e}$ oscillations (dashed line) and $\Delta \mathrm{m}^{2}=1.6 \times 10^{-}$ $2 \mathrm{eV}^{2}$ and $\sin ^{2}(2 \theta)=1.0$ for $v_{\mu} \leftrightarrow v_{\tau}$ oscillations (dotted line).

shows that if matter effects exist in the sun, they should also occur in the earth [36]. Therefore, matter effects, so crucial to our understanding of solar neutrinos, can be in principle tested on earth. If resonance occurs in the sun for neutrino energies of $1 \sim 15 \mathrm{MeV}$, since the density of the earth is $10 \sim 100$ times smaller than at the centre of the sun, we could expect resonance in the earth for neutrino energies from 10 to $1500 \mathrm{MeV}$, which is approximately the energy range of atmospheric neutrinos. 
Neutrino oscillations could also be induced by the gravitational MSW effects [37] in violation of the equivalence principle. These exotic effects will naturally be studied by the new experiments.

Superkamiokande and ICARUS will definitely make an extensive high statistics study of atmospheric neutrinos. ICARUS will detect of the order of 1200 atmospheric neutrino events per year per $5 \mathrm{kt}$ module in $4 \pi$ steradians with high resolution and perfect electron muon recognition. The sensitivity of ICARUS is shown on Figure 21. Superkamiokande will have the advantage of a higher rate with about 3000 events per year.

Matter effects studies will benefit from the possibility in ICARUS to distinguish between neutrinos and antineutrinos. In neutrino interactions, a recoiling proton is associated to the $\mu^{-}$or $\mathrm{e}^{-}$lepton in the final state. In antineutrino interactions, a single lepton is present with some nuclear activity from neutron interactions. In addition, the different capture-to-decay ratio in liquid argon for $\mu^{+}$and $\mu^{-}$allows us to recognize stopping muon charges.

\subsection{Search for $v_{\mu} \leftrightarrow v_{\tau}$ oscillations with $v_{\mu}$ beams}

In the CERN SPS $v_{\mu}$ beam, the relative fraction of $v_{\tau}$ 's produced directly from hadron decays (for instance $p+N \rightarrow D_{S}+$ anything followed by $D_{S} \rightarrow \tau v_{\tau}$ ) should be $\sim 10^{-7}$, allowing in principle detection of $v_{\tau}$ 's produced through $v_{\mu}$ $\leftrightarrow v_{\tau}$ oscillations down to a sensitivity of the same order. The neutrino beam in the West area of CERN has been entirely rebuilt and two new experiments have started data taking in April this year (1994) and the first significant results should be available by the end of 1995 .

WA-95, known as CHORUS [38] from CERN Hybrid Oscillation Research ApparatUS, is using a system of $10^{6}$ scintillating fibres to point tracks of selected events into an emulsion for computer-assisted search for $v_{\tau}$ interactions. The signal for a $\tau$ decay in the emulsion is an apparent 'kink' in, for instance, a muon track extrapolated from the fibre information. The events to be measured are pre-selected with kinematics cuts.

WA-96, known as NOMAD [39] for Neutrino Oscillation MAgnetic Detector, uses a complementary technique based on a very fine-grained target made of 150 drift chamber planes inside the magnet from UA1. The extraction of a $v_{\tau}$ signal is based entirely on kinematics criteria.

The main background is hadrons from the neutral-current process: $v_{\mu}+N \rightarrow$ $v_{\mu}+$ hadrons, faking a $\tau$ decay, and which can be rejected by requiring large 
momentum components $\mathrm{P}_{\tau}>1.6 \mathrm{GeV}$ and $\mathrm{Ph}_{\mathrm{h}}{ }^{-}>1.6 \mathrm{GeV}$ together with topological cuts: the direction of the missing transverse energy in the event must be away from the hadron jet; the lepton or hadron produced in $\tau$ decay must not be back to back with the hadron jet in the azimuthal angle direction. Charm also constitutes a background; the rejection is based on the fact that it is produced in pairs.

CHORUS and NOMAD have similar sensitivities for the mixing parameter which combine to $\sin ^{2}(2 \theta)>2 . \times 10^{-4}\left(90 \%\right.$ C.L.) for $\mathrm{m}_{\nu \tau}>7 \mathrm{eV}$ (Figure 20). The first data taking took place this year and the first results are expected in 1995. The above sensitivity is about 20 times better than the present limit. E803 [40], an approved experiment at Fermilab using the same emulsion technique as CHORUS, should be 10 times more sensitive; however it will not start before the end the decade since it needs the Main Injector.

Another interesting possibility may exist to reach $\sin ^{2}(2 \theta) \sim 10^{-5}$ by using the ICARUS technique with a 100-ton liquid $\mathrm{CH}_{4}$ TPC [36] in a 2-tesla magnetic field. The idea is to use the four quasi-free protons to completely determine the kinematics of $v_{\tau}$-proton collisions from the additional constraint that the incident neutrino direction is known. One could then reconstruct invariant masses and the tau lepton would appear as a mass peak over a very low background reduced by cuts à la CHORUS/NOMAD. This technique would also provide the energy of the incoming neutrino needed to determine the oscillation length, hence the tau neutrino mass. Such a technique, presently under study, would close the window indicated by the MSW interpretation of solar neutrino data.

\subsection{Long baseline neutrino oscillations}

One interesting possibility to increase the sensitivity of accelerator neutrino oscillation experiments is to extend the baseline outside the limits of the laboratory. Long baseline neutrino beam projects are under discussion at CERN, Fermilab, Brookhaven, Serpukhov and KEK. A feasibility study [41] has shown that it is technically possible to send a CERN $v_{\mu}$ beam to Gran Sasso or even to Superkamiokande which both happen to be in favourable azimuthal directions, considering existing or planned beam lines. 


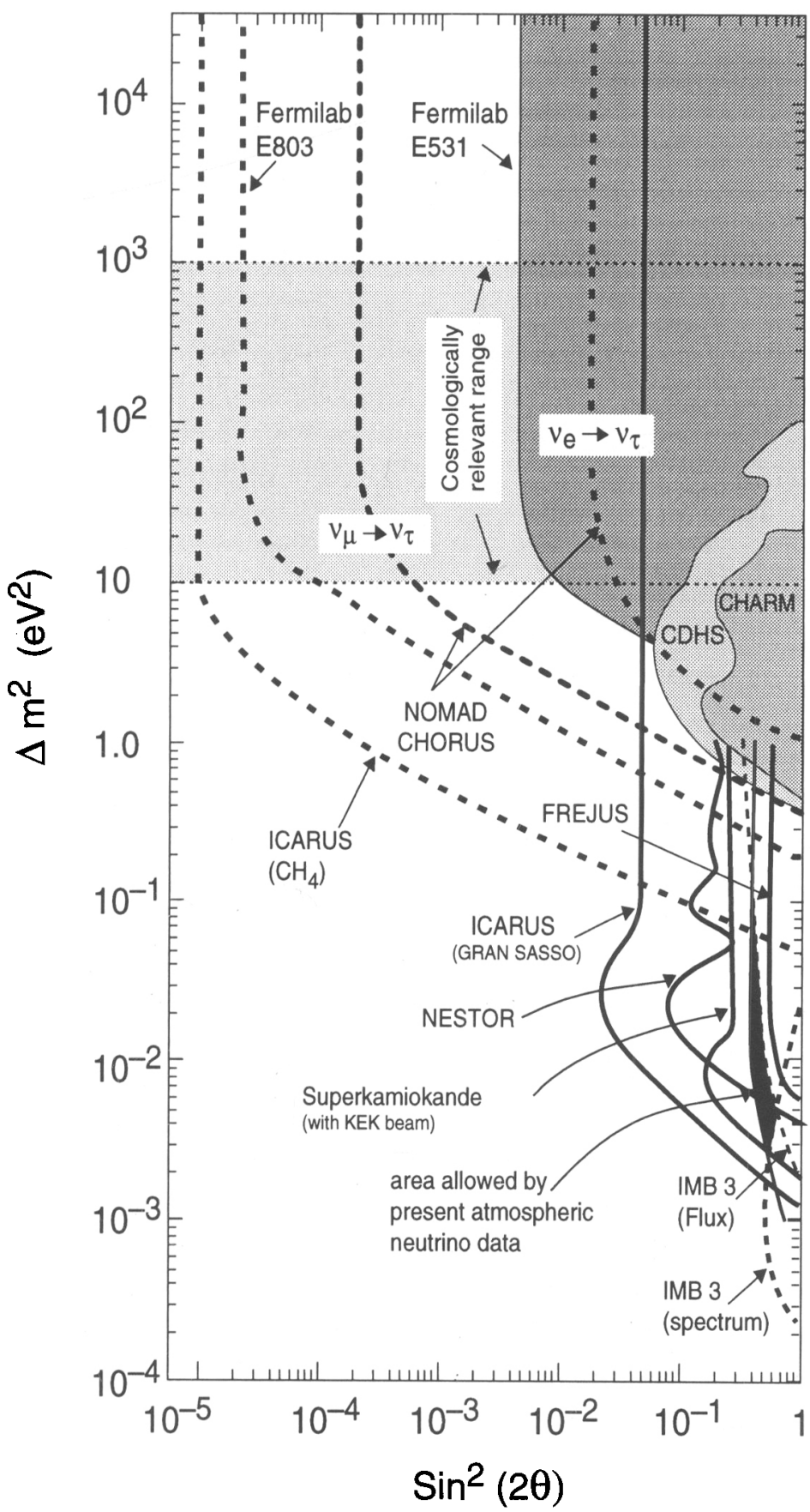

Figure 20: Sensitivity of future $v_{\mu} \leftrightarrow v_{\tau}$ oscillation experiments. 
80

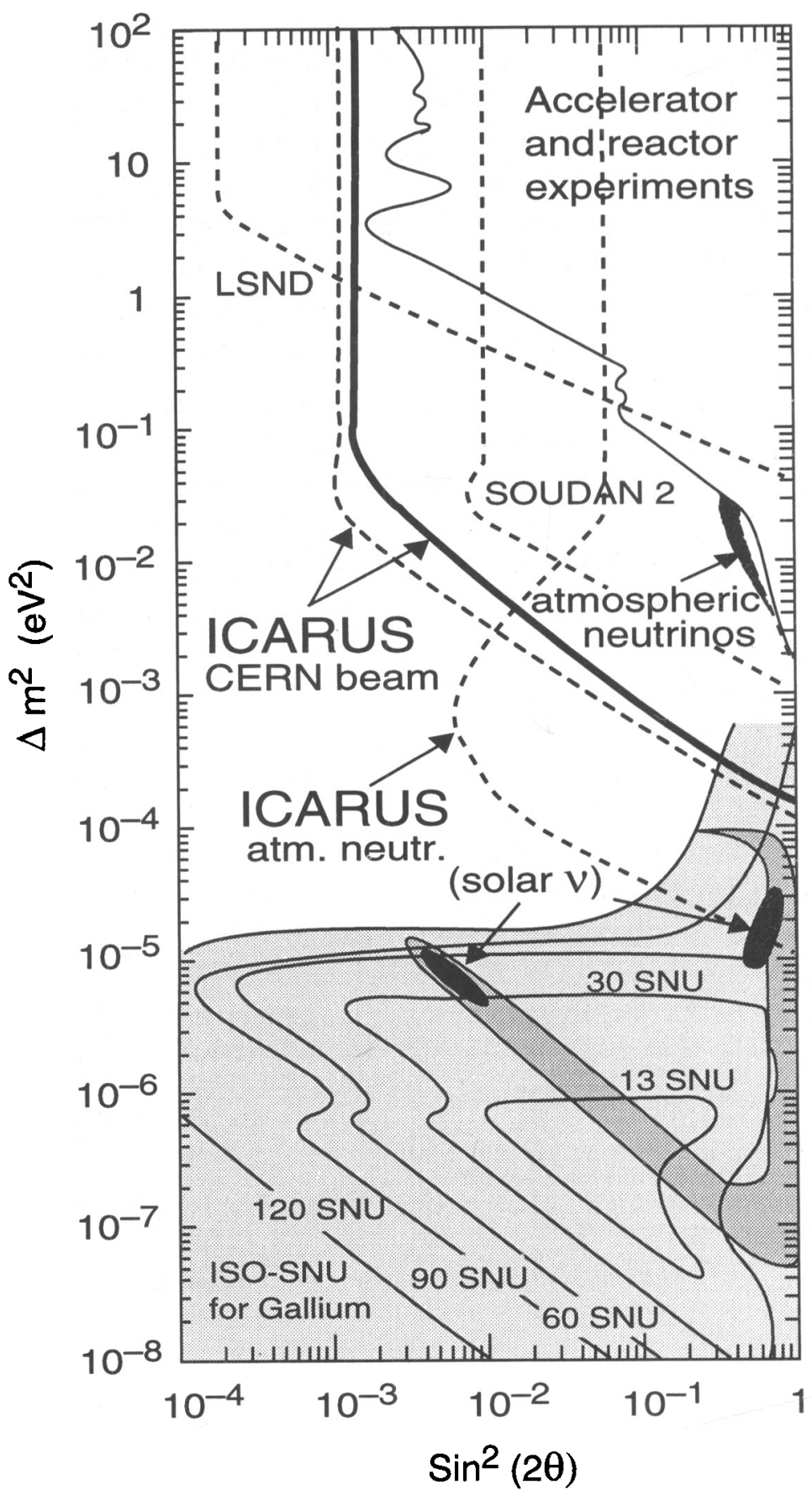

Figure 21: Sensitivity of future $v_{\mu} \leftrightarrow v_{e}$ oscillation experiments. 
Neutrino beams offer several clear advantages: (a) the energy spectrum is known, peaked and can be tuned to the desired range; (b) known direction and timing allow efficient background rejection; (c) high rate for statistical accuracy and known initial beam composition (almost entirely $v_{\mu}$ or $\bar{v}_{\mu}$ ), small $v_{e}$ content at large distances; (d) possibility to switch between neutrinos and antineutrinos (interesting for matter effects); (e) as most neutrino detectors are also proton decay detectors, an accelerator neutrino beam can in principle be used to calibrate the neutrino background to proton decay, this may require running at lower beam energies than envisaged for the standard long baseline physics.

The optimal conditions for $v_{\mu} \leftrightarrow v_{e}$ oscillations with ICARUS and a CERN SPS beam are obtained with a low proton energy $(\sim 120 \mathrm{GeV})$ allowing $3 \times 10^{13}$ protons on target every $3.6 \mathrm{~s}$. There are two main types of background to charged-current interactions of a genuine $v_{e}$ coming from a $v_{\mu}$ oscillation: (a) $v_{e}$ 's in the initial beam: they mostly come from $K$ decays and to a smaller extent from muon decays. Because in $\mathrm{K}$ decays transverse momenta are larger than for $v_{\mu}$ 's coming from $\pi$ decays, as the distance increases, their proportion decreases and at Gran Sasso they are practically negligible for $120 \mathrm{GeV}$ protons $\left(3 \times 10^{-4}\right)$. The contribution from Charm production is also negligible $\sim 10^{-}$

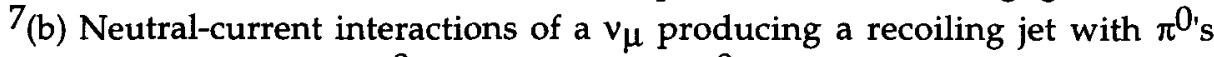
faking electrons $\left(\sim 1.5 \pi^{0} /\right.$ jet $)$. In ICARUS $\pi^{0}$ 's can be identified from their decay properties (separation of the photon showers), from the shape of the shower, and from ionization information. A detailed Monte Carlo simulation has shown that the $\pi^{0}$ background can also be made in practice negligible in the $120-\mathrm{GeV}$ proton case $(\leq 0.1 \%)$. This type of background increases with energy as it becomes harder to identify $\pi^{0} \mathrm{~s}$. The ICARUS sensitivity (Figure 21) extends to $\sin ^{2}(2 \theta)=1.5 \times 10^{-3}$ and $\Delta \mathrm{m}^{2}=1.5 \times 10^{-4} \mathrm{eV}^{2}$.

For the $v_{\mu} \leftrightarrow v_{\tau}$ oscillation study, the highest beam energy is better suited. The CERN accelerator complex could deliver $3 \times 10^{13} 450 \mathrm{GeV}$ protons on target every $7.2 \mathrm{~s}$. The first method consists of measuring the ratio $\mathrm{R}_{\mathrm{A}}$ of neutral current events without a muon in the final state to charged current events with one muon in the final state. One can show that such a ratio can be expressed as: 


$$
R_{A} \approx 0.31\left[1+1.95 \sin ^{2} 2 \theta\left(\Delta m^{2} 1.27 L\right)^{2}\left\langle E^{-2}\right\rangle\right]
$$

where, for a $450 \mathrm{GeV}$ proton beam, we used:

$$
\frac{\sigma_{N C}}{\sigma_{C C}^{\mu}} \approx 0.31 ; \quad f_{\tau}=\frac{\sigma_{C C}^{\tau}}{\sigma_{C C}^{\mu}} \approx 0.5
$$

This so-called appearance method has the merit that it does not require a near detector and it is least sensitive to the magnitude of the $v_{\mu}$ flux, which cancels to first order in the ratio.

A disappearance method can also be used which consists in measuring the ratio of the number of muons produced in the rocks near the detector and coming from the direction of CERN (proportional to the number of $\nu_{\mu}$ 's in the beam) to the total number of neutrino interactions in the detector (which gets contributions from all neutrino flavours).

The combined sensitivity of ICARUS (Figure 20) for the two methods extends to $\sin ^{2}(2 \theta)=5.0 \times 10^{-2}$ and $\Delta \mathrm{m}^{2}=2.0 \times 10^{-3} \mathrm{eV}^{2}$.

There are two main long baseline proposals in the USA. E889 [42], at Brookhaven, is a $v_{\mu}$ disappearance experiment proposing to use a high intensity $1 \mathrm{GeV}$ average energy neutrino beam from the AGS. At Fermilab, P822 [43] is a proposal for a neutrino beam from the Main Injector, aimed at Soudan II, $730 \mathrm{~km}$ away. A comparison [44] between existing simulations of neutrino beams shows that the SPS at CERN and the Main Injector at Fermilab offer equivalent capabilities. These American projects could be completed by the end of this decade. A third American proposal is the GENIUS [45] detector for the Gran Sasso Laboratory.

The long baseline proposal at KEK [46] would rely on an upgraded $12 \mathrm{GeV}$ proton synchrotron, and make use of both a near detector at KEK and Superkamiokande $250 \mathrm{~km}$ away. It is not known at this time whether the project will be approved.

A proposal also exists to send a neutrino beam from UNK at Serpukhov in Russia to the Gran Sasso Laboratory [47], $2200 \mathrm{~km}$ away. 
Matter effects can also in principle be studied with a long baseline neutrino beam, since most of the neutrino path is in the earth. In the case of a CERN beam to Gran Sasso, or a Fermilab beam to Soudan, the neutrino path in matter $(\sim 730 \mathrm{~km})$ is too short to reach a spectacular resonance; however, asymmetry measurements [48] between $v_{\mu}$ and $\bar{v}_{\mu}$ could perhaps allow us to detect the effects. With the Serpukhov neutrino beam to Gran Sasso, if the neutrino flux turns out to be sufficient, the advantage will clearly be an enhanced sensitivity to possible matter effects compared to the CERN or Fermilab neutrino beams [48].

\subsection{Supernova neutrinos}

With the future generation of neutrino detectors in operation we can hope that the next supernova in our galaxy will be observed in the neutrino channel with great details. Superkamiokande would record a thousand $\bar{v}_{e}$ events for a supernova at the galactic centre but with little sensitivity to $v_{e}$ 's from the neutronization burst. The corresponding burst in ICARUS would only produce 76 events ( $44 v_{\mathrm{e}}$ absorption on liquid argon and 32 electron scattering by all six neutrino species). On the other hand ICARUS would record $11 v_{e}$ events from the neutronization burst [23] (Figure 22) and open the possibility of weighing the neutron star produced. In addition, the possibility to observe separately charged and neutral current events as well as identifying neutrino types will result in further sensitivity to neutrino oscillations. Clearly supernovae will offer the longest baseline possible.

Also in the energy window above terrestrial anti-neutrinos and below those of atmospheric origin is the exciting possibility of detecting relic supernova neutrinos whose mean energy is predicted to be around $10 \mathrm{MeV}$.

\section{Conclusion}

Particle physics is assuming the success of the Standard Model. However, the present status of the Standard Model is not a completion point but the start of exciting new ventures. Research in particle physics is very much alive with a future loaded with fundamental questions which often jump from the deep structure of matter to the evolution of the early universe.

From the experimental point of view, all the possible phenomena should be searched for, without too much theoretical prejudice, since most of what I have mentioned concerning future physics is highly speculative. So far: (a) there is no experimental evidence that Supersymmetry is relevant to Nature; (b) our 
dream of unification of forces (GUT), is only driven so far by our hope or belief that Nature meets our criteria for simplicity and beauty; (c) the present experimental situation has room for a lot of improvement, especially in nonaccelerator experiments i.e.: solar and atmospheric neutrinos and proton decay.

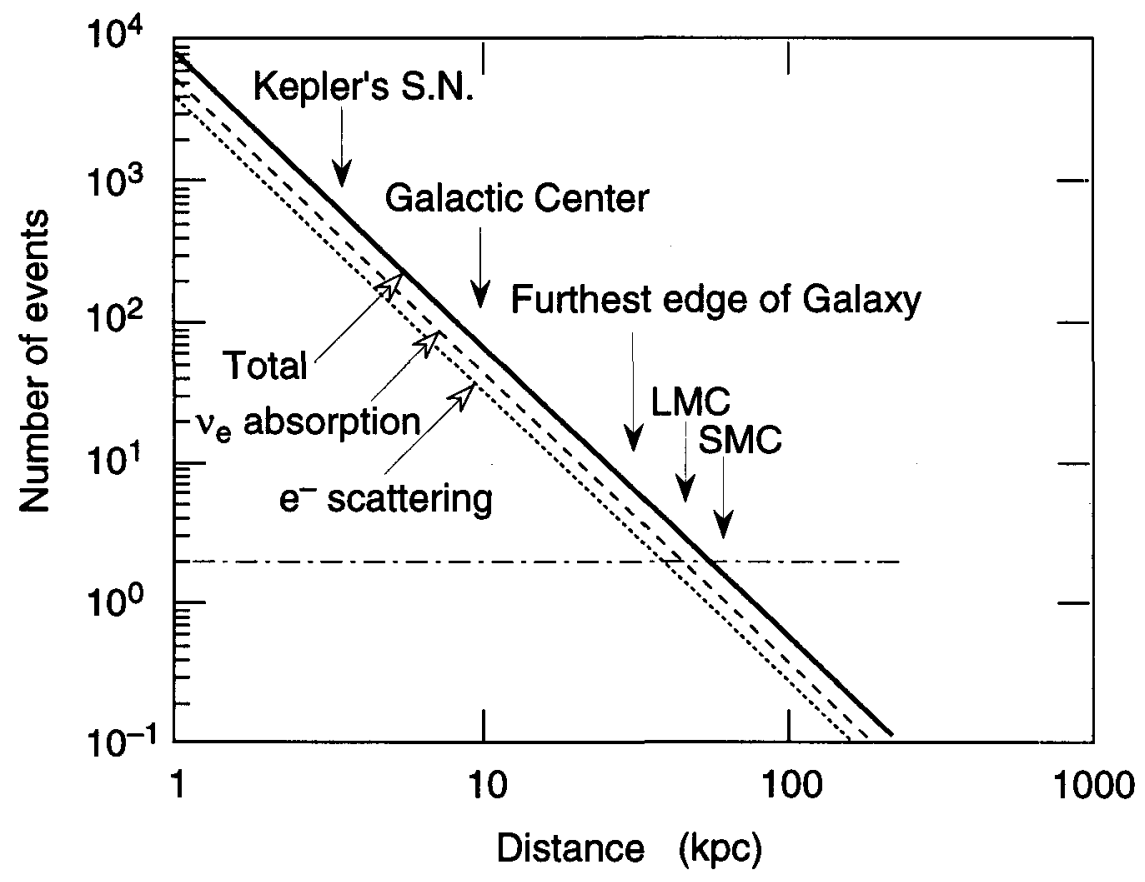

Figure 22: Rate of supernova neutrino events in one 5 kton ICARUS detector module, as a function of distance from the earth and for a total energy release of $2.9 \times 10^{53} \mathrm{erg}$.

For different reasons, both particle physics and cosmology need new forms of matter: (a) particle physics to understand the Higgs mechanism and to achieve unification of forces; (b) cosmology to have enough matter to account for dark matter and eventually confirm that the universe is closed

There are strong indications from our present knowledge that the next generation of particle physics experiments, at the high energy frontier with the LHC, together with neutrino oscillations, proton decay experiments should give us additional answers to some of the extraordinary questions on the nature, past and future of our universe. 


\section{References}

[1] F. Abe et al., "Evidence for Top Quark Production in $\bar{p}$ Collisions at $\sqrt{s}=$ 1.8 TeV", Phys. Rev. Lett. 73 (1994) 225.

[2] F. J. Hasert et al., Phys. Lett. $\underline{46 \mathrm{~B}}, 121$ (1973); F. J. Hasert et al., Phys. Lett. 46B 138 (1973); A. Benvenutti et al., Phys. Rev. Lett. 30, 1084 (1973).

[3] G. Arnison et al., Phys. Lett. 122B, 103 (1983); M. Banner et al., Phys. Lett. 122B 476 (1983).

[4] G. Arnison et al., Phys. Lett. 126B, 398 (1983).

[5] U. Amaldi, W. De Boer and H. Fürstenau, Phys. Lett. 260B 447 (1991); F. Anselmo, L. Ciafarelli, A. Peterman and A. Zichichi, Nuovo Cimento 104A 1817 (1991).

[6] H. Burkhardt, F. Jegerlehner, G. Penso and G. Verzegnassi, in "Polarization at LEP", CERN 88/06; V. A. Novikov, L. B. Okun and M. I. Vysotsky, "On the Effective Electric Charge in the Electroweak Theory", CERN TH 7071/93.

[7] Joint Publication of the four LEP Collaborations, Phys. Lett. 276B 247 (1992); G. Quast, Proc. EPS Conf., Marseille, 1993.

[8] R. Beyer, "Neutrino-Electron Scattering : CHARM II Results", Proc. EPS Conf., Marseille, 1993; G. Râdel and R. Beyer, CERN PPE 93/65, submitted to Mod. Phys. Lett. A.

[9] S. Catani, "QCD and Jets", Proc. EPS Conf., Marseille, 1993.

[10] D. Bufkulic et al. The ALEPH Collaboration, CERN PPE 93/41.

[11] D. Treille, "The LEP 200 Programme", CERN PPE 93/54, to be published in "Precision Tests of the Standard Electroweak Model", World Scientific, 1993, ed. P. Langacker.

[12] L3 Collaboration, "Results from the L3 Experiment at LEP", Phys. Rep. Vol. 236, number 1 \& 2, Dec. 1993.

[13] J. Ellis, "Known and Unknown Matter", Proc. EPS 9, Trends in Physics, Firenze, Sept. 14-17, 1993; M. J. Rees, "The Emergence of Structure in the universe", Proc. EPS 9, Trends in Physics, Firenze, Sept. 14-17, 1993.

[14] R.V. Eötvös, D. Pekar, F. Fekete, Ann. Physik 68 (1922) 11; J. Renner, Mat. Term. Ert. 53 (1935) 542; P.G. Roll, R. Krotkov, R.H. Dicke, Ann. Phys. 26 (1964) 442; V.B. Braginsky, V.I. Panov, Sov. Phys. JETP 34 (1972) 463.

[15] See for instance W. de Boer, "Grand Unified Theories and Supersymmetry in Particle Physics and Cosmology", IEKP-KA/94-01, hep-ph/9402266, March 1994, to be published in Prog. in Part. and Nucl. Phys., and references therein.

[16] U. Amaldi et al., Phys. Rev. D36 (1987) 1385; U. Amaldi, W. de Boer and H. Fürstenau, Phys. Lett. B260 (1991) 447. 
[17] P. Nath, R. Arnowitt, and A.H. Chamseddine, Phys. Rev. 32D, 2348 (1985); J. Hisano, H. Murayama and T. Yanagida, Nucl. Phys. B402, 46 (1993).

[18] R. Arnowitt and P. Nath, Phys. Rev. D49, 1479 (1994).

[19] K.S. Hirata et al., The Kamiokande Collaboration, Phys. Lett. B280 (1992) 146-152.

[20] W. A. Mann, T. Kafka and W. Leeson, Phys. Lett. B291 (1992) 200.

[21] J.L. Thron et al., Soudan II Collaboration, Nucl. Inst. and Meth. 283 (1989) 642.

[22] M. Takita, Proc. Int. Symp. on Neut. Astr., Takayama, Japan, (1992); Y. Totsuka, "Superkamiokande", ICRR-Report-227-90-20, Proc. Int. Symp. on Underground Phys. Exp, Science Council of Japan, April, 1990; K. Nakamura, ICRR-Report-309-94-4, Proc. 3rd NESTOR Int. Workshop, Pylos, Greece, Oct. 1993.

[23] The ICARUS Collaboration, "ICARUS II, a Second-Generation Proton Decay Experiment and Neutrino Observatory at the Gran Sasso Laboratory, LNGS-94/99-I \& 99-II, May 1994.

[24] P. Benetti et al., "Performance of a three-ton Liquid Argon Time Projection Chamber", submitted to Nucl. Instrum. Methods Phys. Res.

[25] S. Bludman et al., Phys. Rev. D45, 1810 (1992); P. G. Langacker and M. X. Luo, Phys. Rev. D44, 817 (1991).

[26] The GALLEX Collaboration, announcement at Gran Sasso, Friday 18th of November 1994.

[27] L. Wolfenstein, Phys. Rev. D17 (1978) 2369, D20 (1979) 2634; S.P. Mikheyev and A.Yu. Smirnov, Yad. Fiz. 42 (1985) 1441; Nuovo Cim. 9C (1986) 17; H. Bethe, Phys. Rev. Lett. 56 (1986) 1305.

[28] D. R. O. Morrison, "The Steady Vanishing of the Three Solar Neutrino Problems", Proc. 27th Int. Conf. on HEP, Glasgow, Scotland, 20-27 July, 1994, CERN-PPE/94-125.

[29] A. Kovetz and G. Shaviv, to be published in Ap. J., May 1994; A. Dar and G. Shaviv, "A Standard Solution to the Solar Neutrino Problem", Technion PH 94-5 and "Has a Standard Model Solution to the Solar Neutrino Problem Been Found?- A Response", Asher Space Research Institute, Israel Institute of Technology, Haifa, 1994.

[30] J. Bahcall et al., "Has a Standard Model Solution to the Solar Neutrino Problem Been Found?", IASSNS-AST 94/13, April 1994, unpublished.

[31] G. T. Ewan et al., "The Sudbury Neutrino Observatory Proposal", SNO-8712, Oct. 1987; H.C. Evans et al., "Scientific and Technical Description of the Mark II SNO Detector", SNO-89-15, Oct. 1989. 
[32] G. Laurenti et al., "A Study of a High Rate Solar Neutrino Detector with Neutrino Energy Determination", CERN/LAA/PC/93-10, Feb. 1993; T. Ypsilantis, "HELLAZ: a High Rate Solar Neutrino Detector with Neutrino Energy Determination", Proc. of The Dark Matter School, Valencia, Spain, 1993. [33] C. Arpesella et al., "Proposal for a Real Time Detector for Low Energy Solar Neutrinos", Vol 1 \& 2, ed. G. Bellini et al., Dept. of Physics, University of Milano, INFN Milano, Italy and R. Raghavan, AT\&T Bell Lab., Murray Hill, NJ, USA.

[34] The Kamiokande Collaboration, "Results from a Test Beam Calibration of a Water Cerenkov Detector at KEK", to be published.

[35] Y. Fukuda et al., "Atmospheric $v_{\mu} / v_{\mathrm{e}}$ Ratio in the Multi-GeV Energy Range", June 1994, presented at the ICHEP 94, Glasgow, to be published.

[36] C. Rubbia, "The Renaissance of Experimental Neutrino Physics", Proc. of Coversaciones de Madrid, Sept. 5, 1992, El Escorial, Madrid, Spain; CERN-PPE / 93-08; J. P. Revol, "Future Neutrino oscillation Experiments", Proc. of Les Rencontres de Physique de la vallée d'Aoste", La Thuile, Aosta valley, Ed. by M. Greco (1993).

[37] M. Casperini, Phys. Rev. D38, 2635 (1988); Phys. Rev. D39, 3606 (1989); A. Halprin and C.N. Leung, Phys. Rev. Lett. 67 (1991) 1833; Nucl Phys. B (Proc. Suppl.) 28A, 139 (19920; I. Kazuhito et al., "A Test of the Equivalence Principle by Long Baseline Neutrino-Oscillation Experiments", TMUP-HEL-9207, Nov. 1992; J. Pantaleone et al., "Neutrino Mixing due to a Violation of the Equivalence Principle", IUHET-228, UDHEP-10-92; J. N. Bahcall et al., "Solar Neutrinos and the Principle of Equivalence", IASSNS-AST 94/54, Oct. 1994, submitted to Phys. Rev. D.

[38] CHORUS Collaboration, N. Armenise et al., CERN-SPSC/90-42 (1990).

[39] NOMAD Collaboration, P. Astier et al., CERN-SPSLC/91-21 (1991), CERN-SPSLC/91-48, SPSLC/P261 Add. 1.

[40] K. Kodama et al., Fermilab P803 Proposal, "Muon Neutrino to tau Neutrino Oscillations", Oct. 1989.

[41] A. Ball, B. Bianchi, J.P. Revol, G. R. Stevenson and E. Weisse, "CERN Beams for Long Baseline Neutrino Oscillations Experiments", CERN-SL/Note 92-75 (BT).

[42] A.K. Mann et al., "Proposal for a Long Baseline Neutrino Oscillation Experiment at the AGS", Brookhaven National Laboratory, Jan. 1993.

[43] W.W.M. Allison et al., Fermilab P822 Proposal, "A Long Baseline Neutrino Oscillation Experiment Using the Soudan II Neutrino Detector", Mar. 1991. 
[44] D. Michael, "Comparison of Long Baseline Neutrino Beam Calculations", California Institute of Technology preprint, 1994 and private communication, to be published.

[45] G.J. Alner et al., "Expression of Interest for a Long Baseline Neutrino Oscillation Experiment Using a CERN Beam and a Large Magnetic Sampling Calorimeter at Gran Sasso", LNGS Proposal, May 1994.

[46] K. Nishikawa, "A Possible Long base Line Neutrino Oscillation Experiment Using KEK PS and Super Kamiokande", INS-Rep.-924, April 1992, submitted to J. of the Phys. Soc. of Japan.

[47] Yu. S. Fedotov et al., "On Possibility to Study Neutrino Oscillations by Detectors Located at Gran Sasso (Italy) Using Beams from 600 GeV UNK-1 Machine", Protvino preprint, January 1994.

[48] G. Fiorentini and B. Ricci, "Old and New Interactions in Long Baseline Neutrino Experiments", INFN Ferrara 06-93, Proc. Workshop on Neutrino Telescopes, Venice, March 1993, ed. M. Baldo-Ceolin.

[49] J.Ellis, G.L. Fogli and E. Lisi, "The top quark and Higgs boson masses in the Standard Model and the MSSM", Phys. Lett. B333 (1994) 118. 